\title{
Starshade rendezvous: exoplanet sensitivity and observing strategy
}

\author{
Andrew Romero-Wolf, ${ }^{a} *$ Geoffrey Bryden $\odot,{ }^{\text {a }}$ Sara Seager $\odot,{ }^{b}$ \\ N. Jeremy Kasdin, $,{ }^{\text {J Jeff Booth, }},{ }^{\text {a }}$ Matt Greenhouse $\odot,{ }^{d}$ Doug Lisman, ${ }^{a}$ \\ Bruce Macintosh, ${ }^{\text {e }}$ Stuart Shaklan $\odot{ }^{\text {a }}$ Melissa Vess, ${ }^{\text {d }}$ Steve Warwick,

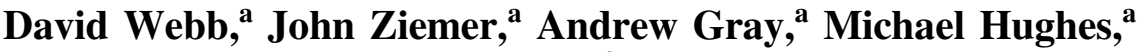

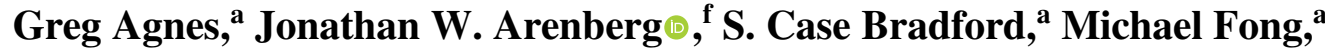 \\ Jennifer Gregory, ${ }^{a}$ Steve Matousek, ${ }^{\text {a Jason Rhodes, }}{ }^{\text {a }}$ Phil Willems, ${ }^{\text {a }}$ \\ Simone D’Amico $\odot$, g John Debes $\odot$, h Shawn Domagal-Goldman $\odot,{ }^{\text {d }}$ \\ Sergi Hildebrandt $\odot$, ${ }^{a}$ Renyu Hu $\odot,{ }^{\text {a }}$ Alina Kiessling, ${ }^{a}$ Nikole Lewis, \\ Maxime Rizzo, ${ }^{\mathrm{d}}$ Aki Roberge, ${ }^{\mathrm{d}}$ Tyler Robinson $\odot$, ${ }^{\mathrm{j}}$ Leslie Rogers $\odot,{ }^{\mathrm{k}}$ \\ Dmitry Savransky ${ }^{\prime}$, and Chris Stark ${ }^{\text {h }}$ \\ aJet Propulsion Laboratory, California Institute of Technology, \\ Pasadena, California, United States \\ ${ }^{\mathrm{b}}$ Massachusetts Institute of Technology, Department of Earth and Planetary Sciences, and \\ Department of Physics, Cambridge, Massachusetts, United States \\ ${ }^{c}$ University of San Francisco, College of Arts and Sciences, San Francisco, \\ California, United States \\ ${ }^{\mathrm{d}}$ NASA Goddard Space Flight Center, Greenbelt, Maryland, United States \\ ${ }^{\mathrm{e}}$ Stanford University, Kavli Institute for Particle Astrophysics and Cosmology, Stanford, \\ California, United States \\ ${ }^{\text {f}}$ Northrop Grumman Aerospace Systems, Redondo Beach, California, United States \\ ${ }^{\mathrm{g}}$ Stanford University, Stanford, California, United States \\ ${ }^{\mathrm{h}}$ Space Telescope Science Institute, Baltimore, Maryland, United States \\ ${ }^{\mathrm{i}}$ Cornell University, Department of Astronomy and Carl Sagan Institute, Ithaca, \\ New York, United States \\ ${ }^{\mathrm{j}}$ Northern Arizona University, Department of Astronomy and Planetary Science, \\ Flagstaff, Arizona, United States \\ ${ }^{k}$ University of Chicago, Astronomy Department, Chicago, Illinois, United States \\ ${ }^{1}$ Cornell University, Sibley School of Mechanical and Aerospace Engineering, Ithaca, \\ New York, United States
}

\begin{abstract}
Launching a starshade to rendezvous with the Nancy Grace Roman Space Telescope (Roman) would provide the first opportunity to directly image the habitable zones (HZs) of nearby sunlike stars in the coming decade. A report on the science and feasibility of such a mission was recently submitted to NASA as a probe study concept. The driving objective of the concept is to determine whether Earth-like exoplanets exist in the HZs of the nearest sunlike stars and have biosignature gases in their atmospheres. With the sensitivity provided by this telescope, it is possible to measure the brightness of zodiacal dust disks around the nearest sunlike stars and establish how their population compares with our own. In addition, known gasgiant exoplanets can be targeted to measure their atmospheric metallicity and thereby determine if the correlation with planet mass follows the trend observed in the Solar System and hinted at by exoplanet transit spectroscopy data. We provide the details of the calculations used to estimate the sensitivity of Roman with a starshade and describe the publicly available Python-based source code used to make these calculations. Given the fixed capability of Roman and the constrained observing windows inherent for the starshade, we calculate the sensitivity of the combined observatory to detect these three types of targets, and we present an overall observing strategy that enables us to achieve these objectives. (1) The Authors. Published by SPIE under a Creative Commons Attribution 4.0 Unported License. Distribution or
\end{abstract}

*Address all correspondence to Andrew Romero-Wolf, Andrew.Romero-Wolf@jpl.nasa.gov 
reproduction of this work in whole or in part requires full attribution of the original publication, including its DOI. [DOI: 10.1117/1.JATIS.7.2.021210]

Keywords: planets; space optics; imaging.

Paper 20113SS received Aug. 1, 2020; accepted for publication Dec. 16, 2020; published online Jan. 9, 2021.

\section{Introduction}

Space-based direct imaging is the next frontier of discovery for exoplanet science, moving beyond detection of Earth-like planets toward full characterization of their atmospheres, potentially identifying signs of life. The Nancy Grace Roman Space Telescope (hereafter referred to as Roman) is scheduled to launch in late 2025. While its Coronagraph Instrument (CGI) will reach planet-star flux ratios only achievable in space, with the capability to directly image gas giant planets around nearby stars, it does not have the sensitivity to detect Earth-like planets. With the launch of a companion starshade, a free-flying 26-m external occulter such a goal can be reached within the next decade.

The Starshade Rendevous Probe (SRP) mission concept was presented in a recent report ${ }^{1}$ assessing the possibility of flying an external starshade. Using the CGI, the starshade significantly enhances the exoplanet observing capabilities of Roman by providing (1) improved contrast and a reduced inner working angle (IWA), enabling sensitivity to Earth-like exoplanets in the habitable zone (HZ); (2) an unlimited outer working angle, enabling a wider view of the planetary system around each target; and (3) higher throughput (the coronagraph masks are not needed with a starshade), enabling the sensitivity needed to spectrally characterize an Earth-like exoplanet.

In this paper, we provide the technical basis for the SRP study report ${ }^{1}$ along with the simulations used to estimate sensitivity of the observatory. While many such assessments have been done in the past, ${ }^{2-5}$ this study started by defining the science objectives of the mission with detailed knowledge of Roman constraints, the coronagraph instrument (CGI) expected performance, and the significant advances of NASA's S5 Starshade technology development program. ${ }^{6}$ While detailed simulations of Starshade missions exist, ${ }^{5,7,8}$ we found that the SRP has a relatively small number of targets, which imposes different demands. The need to understand how to balance the different objectives of the mission, within significant mission constraints, and how to prioritize targets led us to produce a simulations package focused on the SRP. This software is publicly available for reproduction of the results below and for comparison with similar simulations (https://github.com/afromero/Starshade_Rendezvous_ Probe_sims).

The paper is structured as follows. We briefly review the science objectives presented in the SRP report (Sec. 2), followed by an outline of the top-level mission constraints based on its partnering with the Roman (Sec. 3). We then describe the model used to quantify the starshade performance, both the observatory characteristics and the expected astrophysical scene (Sec. 4). Based on this observing plan, we next calculate the expected integration times required to achieve a given signal-to-noise (SNR) given Roman's nominal optical design (Sec. 5). We use the observing model to calculate the sensitivity of the observatory toward individual targets (Sec. 5) and then provide an overall observing strategy, including choices on the number of stars, the number of visits per star, and when to switch from imaging to spectroscopy (Sec. 6). Last, in Sec. 7, we calculate the expected performance of the mission, with a specific list of target stars and summarize in Sec. 8.

\section{Science Objectives}

The Starshade Rendezvous Probe Mission Study Report ${ }^{1}$ developed both the scientific motivation and technical feasibility for such a mission. Further detail on the science case can be found there; here, we focus not on motivating the science goals, but on whether these goals can be achieved. 
In summary, the science objectives of the mission are threefold:

- Objective 1: Habitability and Biosignature Gases and the Nearest Solar System (SS) Analogs. (a) Determine whether Earth-like exoplanets exist in the HZs around the nearest sunlike stars and whether have they have signatures of oxygen and water vapor in their atmospheres. (b) Detect and characterize planets orbiting the nearest sunlike stars.

- Objective 2: Brightness of Zodiacal Dust Disks. Establish if the zodiacal cloud of our inner $\mathrm{SS}$ is representative of the population of our nearest neighbor stars.

- Objective 3: Gas-Giant Atmospheric Metallicity. Determine the atmospheric metallicity of known cool giant planets to examine trends with planetary mass and orbital semi-major axis, and determine if these trends are consistent with our SS.

The first objective, finding Earth-like planets, is paramount. As such, maintaining the sensitivity to discover and potentially characterize Earth-like exoplanet candidates around these stars drives the observatory requirements. Meeting these challenging requirements means that the observatory will also be capable of discovering and characterizing a wide range of planet types, from those similar to the giant planets in our SS to the sub-Neptune planets commonly discovered by Kepler ${ }^{9}$ down to Earth-mass planets.

The difficulty of imaging Earth-like planets necessitates a deep dive approach, i.e., a detailed investigation of a relatively small sample of our nearest-neighbor sunlike stars-only the closest sunlike stars where starshade observations have both high imaging sensitivity for exoplanet discovery and high spectral sensitivity to characterize their atmospheric composition, while also allowing multiple visits to constrain the orbits of any habitable-zone planets found (see Ref. 1 for a more detailed discussion). Planetary systems almost certainly exist around several of these stars. ${ }^{10}$ If an Earth-like planet candidate is discovered around at least one of these stars, it will be spectroscopically observed to hunt for water vapor and oxygen. Three steps are needed to accomplish this goal: (1) initial detection via direct imaging; (2) HZ determination by multiepoch orbit tracing; and (3) atmosphere characterizatio, with spectroscopy of the most compelling candidate planets, particularly those in the HZ. Each of these steps places unique requirements on the mission parameters, as described in this paper.

The requirement to detect Earth-like exoplanets also enables the ability to detect exozodiacal dust disks and spectral features of gas giants. The distribution of exozodiacal dust brightness, at the level relevant for Earth-like exoplanet detection, is largely unconstrained. Recent bounds on the warm dust disk brightness of many sunlike stars of interest to the SRP are provided by large binocular telescope interferometer (LBTI), ${ }^{11}$ but the sensitivity is still more than an order of magnitude in excess of what is needed. Objective 2 will provide the key information necessary to assess the sensitivity to directly image $\mathrm{HZ}$ exoplanets.

Objective 3 will test whether the correlation between atmospheric gas metallicity and planet mass (as well as semi-major axis) observed in our SS, and hinted at in a few transit spectroscopy measurements of exoplanets, ${ }^{12}$ is a universal trend. Identifying this correlation with the SRP would provide evidence of common processes of planetary system formation.

\section{Mission Constraints}

The Starshade Rendezvous Probe is an enhancement of the Roman mission. As such, many of the starshade mission's parameters are fixed by Roman's established telescope, instruments, and operational timeline. The key constraints imposed on a starshade mission are summarized in Table 1 (It is worth noting that all of the parameters in Table 1 are up-to-date at the time of this study. A full list of current Roman coronagraph characteristics can be found at Ref. 13). In particular, Roman's CGI will be used for imaging and spectroscopy. The bandpass and spectral resolution for this instrument are designed for a similar science case (exoplanet imaging/ spectroscopy) and as such are a good match to our science goals.

The field of regard is limited by both the telescope and the starshade. Roman cannot point within $54^{\circ}$ of the Sun, or light will scatter into the telescope assembly. Solar angles $>83^{\circ}$ are excluded by the starshade; beyond this limit, the starshade can no longer be viewed close to face on without being illuminated by sunlight. Although this last point is not technically an imposed 
Table 1 Mission constraints.

\begin{tabular}{lc}
\hline \hline Parameter & Expected performance \\
\hline Starshade nominal mission lifetime & 2 years \\
Telescope primary mirror & $2.4 \mathrm{~m}$ \\
Solar exclusion angle (min) & $54^{\circ}$ \\
Solar exclusion angle (max) & $83^{\circ}$ \\
Detector bandpass & 400 to $1000 \mathrm{~nm}$ \\
Imaging resolution & 65 mas at $750 \mathrm{~nm}$ \\
Imaging end-to-end efficiency & $0.035^{\mathrm{a}}$ \\
Imaging FOV & $4.5^{\prime \prime}$ (radial) \\
\hline \hline
\end{tabular}

aSee Table 2 for details.

constraint, it is an important one imposed by the Starshade architecture and limits the target observing windows.

The imaging end-to-end efficiency, discussed in more detail in Sec. 5, is the result of losses from the telescope aperture, optical path, with coronagraph masks excluded, and all the way down to the quantum efficiency $(\mathrm{QE})$ of the detector. The Starshade observations depend on the CGI camera with little modifications, so we take this as an imposed constraint. The field of view (FOV) of the CGI detector is limited to 4.5" (radial). This parameter is important for detecting any planets orbiting outside the $\mathrm{HZ}$ of the target of interest.

Lastly, it is worth noting that, while Roman's lifetime requirement is five years, we assume the starshade launch will occur three years into the mission, giving an overlap of two years. Extending beyond that duration is ultimately limited by the lifetime of the CGI camera and the overall Roman system.

\section{Observing Model}

This section covers the observing model used to calculate the SNR of an exoplanet directly imaged with a telescope-starshade system. The overall observing model is shown as a flowchart in Fig. 1. This model consists of both astrophysical inputs, shown in the red boxes, (Sec. 4.1) and instrument parameters, shown in the green boxes (Sec. 4.2), which are combined to estimate the SNR (Sec. 4.3).

The exoplanet direct imaging observing geometry of the telescope-starshade system is shown in Fig. 2. The starshade blocks the starlight up to an IWA, above which exoplanets can be observed. A planet at radius $R_{p l}$ is observed with illumination phase angle $\beta$. For $\mathrm{HZ}$ exoplanets, defined by the inner habitable zone (IHZ) and outer habitable zone (OHZ) radii where water can exist in its liquid phase, the telescope-starshade system has a region of allowed Sun angles over which it can operate with the lower limit defined by the exclusion angle from the baffle of the telescope and the outer limit defined by reflection and scattering of sunlight off the starshade into the telescope baffle.

\subsection{Astrophysical Model}

The signal is produced by the star's flux density being reflected off the surface of the planet. The astrophysical sources of background light are the exozodiacal dust disk and our own SS's zodiacal dust. For this section, we focus solely on the astrophysical model with the parameters provided in Sec. 5.1.1. 

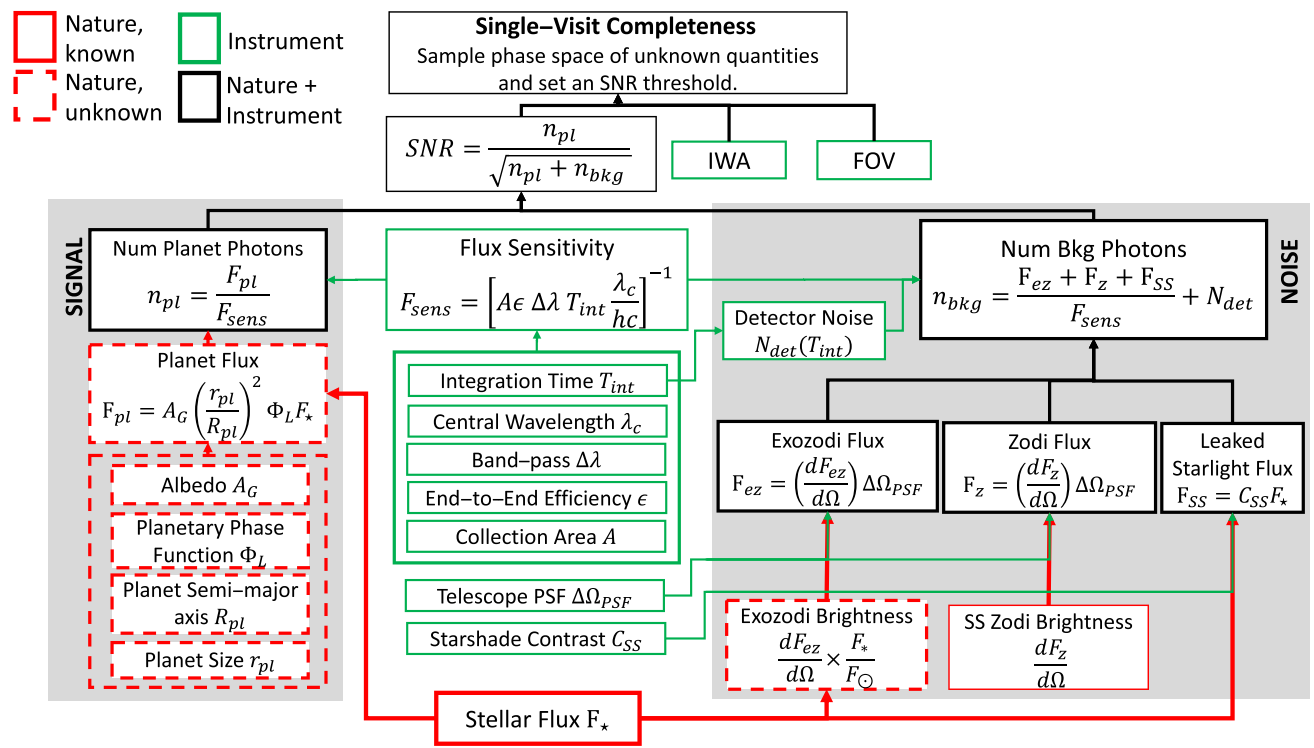

Fig. 1 The detailed observatory model used for the sensitivity estimates in this study. Single-visit completeness is determined by IWA, FOV, and SNR resulting from a planet observed with the SRP system. Red boxes are parameters entirely determined by nature, green boxes are parameters controlled by observatory design, and black boxes are a combination of both. The same model is used for spectral completeness using the corresponding values for bandpass and end-to-end efficiency.

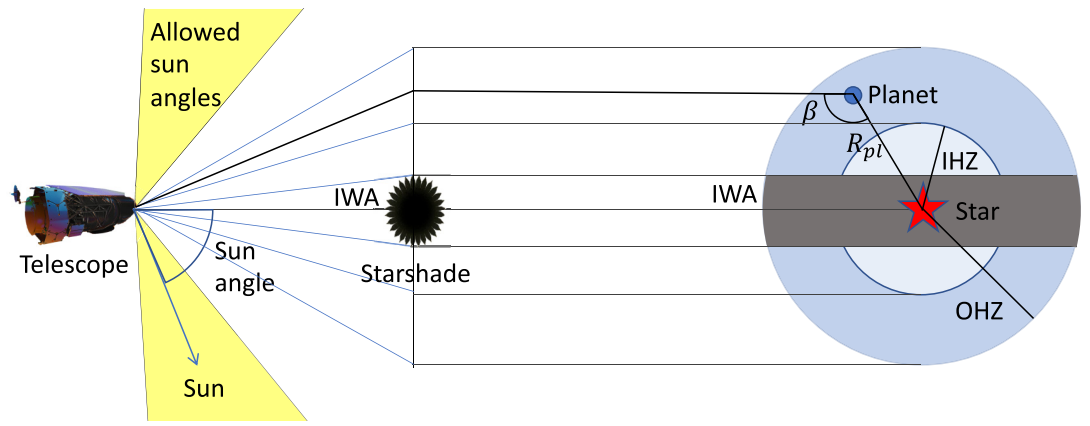

Fig. 2 Starshade observatory geometry. The telescope is pointed at a star with the starshade blocking the starlight. The IWA is the angle subtended by the direction to the star and the outer radius of the starshade. Observations of planets in this region are excluded. The $\mathrm{HZ}$ of the star is defined by the $\mathrm{IHZ}$ radius and $\mathrm{OHZ}$ radius, defined by the distance to the star where the stellar irradiance allows for water in liquid state. A planet at radius $R_{p l}$ has illumination phase angle $\beta$ defined as the star-planet-telescope angle. The telescope-starshade system has a region of Sun angles at which it can observe. The lower limit is set by the solar exclusion angle of the telescope baffle while the upper limit is set by reflection of the Sun off the starshade's surface on the telescope. These solar exclusion angles result in important observational constraints.

\subsubsection{Star flux density}

We use a simple black body model for the star. The model inputs are the bolometric luminosity $L_{\star}$, blackbody temperature $T_{\star}$, stellar mass $M_{\star}$, and distance from Earth $d_{\star}$. The star's spectral radiance is given by Planck's law

$$
B_{\star}\left(\lambda, T_{\star}\right)=\frac{2 h c^{2}}{\lambda^{5}} \frac{1}{\exp \left(h c / \lambda k T_{\star}\right)-1} .
$$


The flux density $F_{\star}$ at Earth in a band defined by limits $\lambda_{\min }$ and $\lambda_{\max }$ is given as

$$
F_{\star}=\frac{L_{\star}}{4 \pi d_{\star}^{2}} \frac{1}{\sigma_{\mathrm{SB}} T_{\star}^{4}} \int_{\lambda_{\min }}^{\lambda_{\max }} \mathrm{d} \lambda B\left(\lambda, T_{\star}\right),
$$

where $\sigma_{\mathrm{SB}}$ is the Stefan-Boltzmann constant. The first fractional term in the equation is the total wavelength-integrated flux. The second fractional term is the normalization by the StefanBoltzmann law, which is the integral of Eq. (1) over all wavelengths.

The input data for the stars used in this study were obtained from ExoCat-v1 (M. Turnbull (2015), "ExoCat-1: The Nearby Stellar Systems Catalog for Exoplanet Imaging Missions," arXiv: $1510.01731,{ }^{14}$ which is a compilation of stars within $30 \mathrm{pc}$. The target selection is discussed in Sec. 5.

\subsubsection{Planet flux density}

For bodies that are not self-luminous, the flux density depends entirely on the light reflected from its surface by the host star with flux density $F_{\star}$. The fraction of light reflected depends on the ratio of the projected disk area of the planet with radius $r_{\mathrm{pl}}$ and square distance from the star $R_{\mathrm{pl}}$. The dependence of the reflected light on the phase angle $\beta$ (i.e., the planet-star-observer angle) is assumed to be Lambertian

$$
\Phi_{L}(\beta)=\frac{\sin \beta+(\pi-\beta) \cos \beta}{\pi} .
$$

This assumption of isotropic scattering is approximately correct for cloudy gas giants, ${ }^{15,16}$ but not for rocky planets that have enhanced forward scattering. Our assumed geometric albedo for Earth-like planets (0.2) only matches the Earth's reflectivity for scattering angles $\beta \lesssim 90^{\circ}$; for more grazing angles, it is an underestimate. ${ }^{17,18}$ The function $\Phi_{L}(\beta)$ is normalized to unity at maximum illumination $(\beta=0)$, takes the value of $1 / \pi$ at maximum elongation $(\beta=\pi / 2)$, and vanishes smoothly as the planet eclipses the $\operatorname{star}(\beta \rightarrow \pi)$. The normalization of the reflected light at maximum illumination, taking into account the $\left(r_{\mathrm{pl}} / R_{\mathrm{pl}}\right)^{2}$ dependence and $\Phi_{L}(\beta)$, is the geometric albedo $A_{G} \cdot{ }^{19}$ Altogether, the flux of a planet is given as

$$
F_{\mathrm{pl}}(\beta)=A_{G}\left(\frac{r_{\mathrm{pl}}}{R_{\mathrm{pl}}}\right)^{2} \Phi_{L}(\beta) F_{\star} .
$$

The model above applies to the entire range of planet types considered in this study (Earths, Super-Earths, Subneptunes, Neptunes, and Jovian) with different parameters. The parameters used for this study are provided in Sec. 5.

\subsubsection{Dust}

The main sources of natural backgrounds are the sunlight scattered by zodiacal dust within our own SS and the starlight scattered by exozodiacal dust surrounding the exoplanet. Our model for the SS zodiacal dust is from Ref. 20, which considers variations with both wavelength and direction; the ecliptic latitude and longitude of each target star are taken into account. The exozodiacal dust brightness for a 1-zodi disk is set to $22 \mathrm{mag} / \mathrm{arc} \mathrm{sec}^{2} .^{21}$ This is somewhat higher than the nominal brightness of the SS zodiacal dust itself ( $\left.23 \mathrm{mag} / \mathrm{arc} \mathrm{sec}^{2}\right)$ because the exozodiacal dust is twice as thick (we only look through half of the SS zodiacal dust's thickness) and because we view it at more forward-scattering angles. ${ }^{4}$ While some of our target stars do have measured levels of exozodiacal dust, most are non-detections. Analysis of these upper limits suggests a median dust thickness a factor of 4.5 higher than that of the SS. ${ }^{11}$ We adopt this enhanced level as our fiducial amount of exozodiacal dust. The exozodi model is scaled based on the ratio of stellar flux to solar flux and corrected for orbital location relative to Earth-equivalent insolation distance (EEID) given by $1 \mathrm{AU} \times\left(L_{\star} / L_{\odot}\right)^{1 / 2}$. 
The flux density of SS zodiacal and exozodiacal dust backgrounds are proportional to the solid angle subtended by the point spread function (PSF) core $\left(\Delta \Omega_{P S F}\right)$, which is inversely proportional to the telescope diameter. For a brightness distribution $d F / d \Omega$, the flux density is approximated as

$$
F=\left(\frac{d F}{d \Omega}\right) \Delta \Omega_{P S F}
$$

\subsection{Observatory Model}

The observatory is a combination of the Roman telescope, including the CGI instrument, and starshade occulter.

\subsubsection{Telescope}

The sensitivity of the telescope can be summarized in a single value $F_{\text {sens }}$, which is the flux that would produce a single photon count on average for a given integration time $T_{\mathrm{int}}$. This is given as

$$
F_{\text {sens }}=\left[A \epsilon \Delta \lambda T_{\mathrm{int}} \frac{\lambda_{c}}{h c}\right]^{-1},
$$

where $A$ is the geometric aperture of the telescope, based solely on its diameter, and $\epsilon$ is the endto-end efficiency, which fully accounts for the fraction of photons entering the geometric aperture that produces photon counts in the detector. The product $\epsilon A$ is the effective area of the telescope. The bandwidth is given by $\Delta \lambda$ with $\lambda_{c}$ being central wavelength, $h$ is the Planck's constant, and $c$ is the speed of light. The detector noise produces a photon-equivalent background count rate given by $N_{\mathrm{det}}$. The model described in the rest of this section relies heavily on the models used for CGI. ${ }^{22,23}$

Roman is a 2.4-m diameter optical telescope, corresponding to a collecting area of $A=4.5 \mathrm{~m}^{2}$. The diameter spatial full-width at half maximum resolution is $\theta_{\mathrm{PSF}}=0.065^{\prime \prime}$ at $750 \mathrm{~nm}$. The pixel scale for the CGI instrument is $0.0218^{\prime \prime}$. The solid angle subtended by the PSF is approximated as $\Delta \Omega_{P S F} \simeq \pi \theta_{\mathrm{PSF}}^{2}$, which gives $\Delta \Omega_{P S F} \simeq 3.1 \times 10^{-13}$ sr at $750 \mathrm{~nm}$.

The Starshade imaging bandpass filter $(615$ to $800 \mathrm{~nm})$ is tuned to be sensitive to water vapor and oxygen absorption lines at 720 and $760 \mathrm{~nm}$, respectively. The imaging FOV of 4.5" (imaging) enables observations well beyond the HZs of the nearest sunlike stars, providing the potential for discovery of giant outer planets.

The focal plane detector is an electron-multiplying CCD (EMCCD). The detector noise is $\sim 10$ counts $/ \mathrm{h}$, including noise equivalent dark current (dominant term), clock induced charge, and read noise (negligible contribution). While the EMCCD detector has the advantage of no read noise, it is significantly degraded by cosmic ray hits; five years of degradation is included, reducing the detector $\mathrm{QE}$ to $28.5 \%$. $^{3}$

The end-to-end efficiency $(\epsilon)$, which includes the optical throughput of the telescope and the detector quantum efficiency, is 3.5\% for imaging and 3.4\% for spectroscopy. A complete budget for the factors going into these throughput calculations is given in Table 2. The Roman pupil has a central obscuration that results in a reduction of the raw collecting area by $18 \%$. The light collected at the aperture goes through multiple reflections in various elements of the telescope optics to deliver it to the CGI with a further reduction of $19 \%$. Prior to reaching the CGI, a dichroic beam splitter divides the signal into the CGI and a guidance camera with $90 \%$ efficiency. Within the CGI, there are multiple optical elements prior to delivering the light to the detector with an efficiency of $60 \%$. The detector effective $\mathrm{QE}$ is a combination of effects, including QE, cosmic rays, and other detector effects, of $28.5 \%$. We use the end-of-life value since the starshade would operate in the last couple of years of the Roman telescope. The PSF has $34 \%$ of its total light in the core due to the diffraction from the struts and central obscuration region of the Roman aperture. The top-level instrument characteristics are summarized in Table 1. 
Table 2 Telescope efficiency $(\epsilon)$.

\begin{tabular}{lc}
\hline \hline Contribution & Best estimate \\
\hline Geometric obscuration of the WFIRST pupil & 0.82 \\
Reflection losses in the telescope optics & 0.81 \\
Reflection and transmission losses (excluding coronagraph masks) & $0.60(\mathrm{P}) 0.58(\mathrm{~S})$ \\
Starshade dichroic beam splitter & 0.90 \\
Detector effective QE (at end of life) & 0.285 \\
Core throughput losses due to diffraction from WFIRST pupil & 0.34 \\
Total & $0.035(\mathrm{P}) 0.034(\mathrm{~S})$ \\
\hline \hline
\end{tabular}

Note. $-\mathrm{P}=$ Photometry; $\mathrm{S}=$ Spectroscopy

While the model in the previous section provides an adequate description for the wide-band imaging mode, the spectrograph requires some additional details. The currently planned implementation is a slit-prism spectrograph, which blocks all but a narrow region with a slit of width $D$ and disperses the light orthogonal to the slit's long axis. The key parameter for the design is the slit width $D$, which must be wide enough to accommodate for telescope jitter and motion of the planet during a long period of observation while not being so wide that it allows for a significant amount of zodiacal and exozodiacal dust brightness background photons to disperse into the pixels of interest for the exoplanet. The prism-detector configuration is described by the spectral resolution parameter $R=\lambda / \Delta \lambda$, which can, in general, be wavelength dependent. It is worth noting that the spectrograph slit limits observations to one planet at a time, but still allows for simultaneous measurement of the background along the slit.

\subsubsection{Starshade}

The starshade performance can be summarized by three key parameters - the IWA, the instrument contrast $\left(C_{S S}\right)$, and the solar exclusion angles (see Table 3 ).

The starshade IWA is the angle subtended by the telescope boresight and the outer radius of the starshade. The starshade design considered here has $\sim 100 \%$ optical throughput at the IWA. ${ }^{24}$ Although it is, in principle, possible to observe targets at angles below the IWA, the throughput is reduced and the PSF is distorted. For practical purposes, we assume exoplanets are only observable at angles above the IWA. The size of the starshade and the distance between the starshade and telescope are determined primarily by the desired IWA, the longest wavelength in the observing band, the diameter of the shadow at the telescope, and the required suppression level. The optical bandwidth, constraints on feature sizes, and factors such as the ratio of the petal length to the overall diameter and the number of petals are examples of other factors that also impact the size and separation of the starshade, see Refs. 25-28). The IWA is chosen to observe the HZs of nearby sunlike stars. An IWA of 100 mas corresponds to a separation of 1 AU at $10 \mathrm{pc}$, enabling observations of Earth-like planets for solar-type stars within $\sim 10 \mathrm{pc}$.

Table 3 Assumed mission parameters.

\begin{tabular}{lc}
\hline \hline Parameter & Assumed performance \\
\hline Time allocation $^{1}$ & 136 days \\
IWA & $100 \mathrm{mas}$ \\
Instrument contrast & $4 \times 10^{-11}$ \\
Delta-V for retargeting & $1100 \mathrm{~m} / \mathrm{s}$ \\
\hline \hline
\end{tabular}


The starshade instrument contrast $\left(C_{S S}\right)$ is defined as the fraction of starlight leaked per resolution element at the IWA. The resulting background flux is $F_{S S}=C_{S S} F_{\star}$. This results in a background contribution in the $\mathrm{HZ}$ with $C_{S S}$ specified so as to keep it below the expected zodi and exozodi backgrounds, enabling the sensitivity to detect Earth-like exoplanets. The current best estimate for the contrast is $C_{S S}=4 \times 10^{-11}$ (see Ref. 1 for details).

In addition to scattered starlight, solar glint and diffracted speckle patterns from the target result in an additional localized, predictable backgrounds that can be calibrated. While solar glint can be a limiting factor, recent progress in petal edge design indicates that it can be suppressed outside the IWA to negligible levels. We, therefore, do not include a treatment of solar glint in this study; for a more detailed treatment see Ref. 29. The starshade speckle pattern and its ability to remove it during analysis are not included in this study. It is expected that they become an important source of backgrounds. These effects will be accounted for in more detail with more recent tools such as the SISTER simulations package and upcoming Starshade data challenges.

The starshade can accommodate a relatively wide bandpass (26\%), compatible with the bandpass of the CGI (Table 1). The relation between starshade design and bandpass is discussed in Ref. 26.

One important observing constraint is that, with a starshade, the telescope pointing is limited to at most $83^{\circ}$ from the Sun. Beyond this solar exclusion angle, the starshade reflects a significant amount of sunlight into the telescope.

\subsection{Signal-to-Noise Ratio Model}

The $S N R$ is approximated as a function of the number of photon counts from the planet of interest $n_{\mathrm{pl}}$ and the sum total contribution of background photon counts $n_{\mathrm{bkg}}$ according to

$$
S N R=\frac{n_{\mathrm{pl}}}{\sqrt{n_{\mathrm{pl}}+n_{\mathrm{bkg}}}} .
$$

The conversion of the planet flux $F_{\mathrm{pl}}$ to photon counts $n_{\mathrm{pl}}$ is given by the observatory's single-photon flux sensitivity $F_{\text {sens }}$ [Eq. (6)] according to $n_{\mathrm{pl}}=F_{\mathrm{pl}} / F_{\text {sens }}$.

The background photon counts, $n_{\mathrm{bkg}}$, have two main contributions, the external background fluxes (leaked starlight, zodiacal, and exozodiacal light) and the detector noise counts $N_{\text {det }}$. The external background fluxes are converted to background photon counts via the relation to the observatory's single-photon flux sensitivity to give

$$
n_{\mathrm{bkg}}=\frac{F_{e z}+F_{z}+F_{S S}}{F_{\text {sens }}}+N_{\text {det }} .
$$

The modifications to the SNR estimate for spectroscopy are listed as follows. The bandwidth now corresponds to each sub-band of the spectrometer, which is given by $\Delta \lambda=\lambda / R$. With a characteristic value of $R \sim 50$, the bandwidth at the central wavelength of $750 \mathrm{~nm}$ is $15 \mathrm{~nm}$ instead of $185 \mathrm{~nm}$. This reduces the single-photon flux sensitivity $F_{\text {sens }}$ by roughly an order of magnitude compared with imaging mode.

The leaked starlight and the zodiacal and exozodiacal emission can be dispersed into the planet spectrum. If $\theta_{\text {slit }}$ is the angular width of the slit, the effective increase in the background photon counts $n_{\mathrm{bkg}}$ is a factor of $\theta_{\text {slit }} / \theta_{P S F}$

$$
n_{b k g}=\frac{\theta_{\text {slit }}}{\theta_{P S F}} \frac{F_{e z}+F_{z}+F_{S S}}{F_{\text {sens }}}+N_{\text {det }}
$$

under the assumption that $\theta_{\text {slit }} \geq \theta_{P S F}$. A slit width of 120 mas is assumed for the Starshade slit prism spectrometer. The reason the slit has to be wider than the PSF core of 65 mas is to accommodate the a priori unknown motion of an Earth-like exoplanet over the data lag of several days nominal spectral integration time period of 25 days. We are assuming that, during a spectroscopic observation, the slit position can be adjusted over a period of several days given data telemetry, analysis, and commanding latencies. 
The estimates made here assume that the leaking starlight, stray light from the starshade, and exozodi light have been approximated using smooth distributions. The treatment of deviations from these assumptions requires more sophisticated imaging simulation tools (such as SISTER) and exploring a wider range of second-order corrections to be considered. These will be treated in future mission concept studies.

\section{Target Sensitivity}

The analysis presented in this section estimates the performance of the observatory on a pertarget basis and has not assumed any constraints on retargeting time or total mission duration, which are covered in the next section. This serves as an initial bound on how many targets the observatory is sensitive to and sets a clear goal for the more complicated problem of visit strategy and retargeting maneuvers. We divide the discussion between three types of targets, corresponding to the three objectives described in Sec. 2: (1) potential Earth-like planets orbiting bright nearby stars (Sec. 5.1), (2) known exoplanets from radial velocity measurements at wide angular separation from their host star (Sec. 5.2), and (3) dust disks that may surround any of the target stars (Sec. 5.3).

\subsection{Sensitivity to Earth-Like Planets}

\subsubsection{Parameters}

The parameters that define the Star's flux density [Eq. (2)] and mass are taken from ExoCat. ${ }^{30}$ Table 5 shows the star parameters for targets selected by the procedure defined later in this section. The terrestrial planet parameters in our model [Eq. (4)] are assumed to have an Earth-like geometric albedo of $A_{G}=0.2$, based on distant observations of Earth with the EPOXI spacecraft, ${ }^{19}$ along with the Lambertian (isotropic) scattering phase function in Eq. (3). The range of planet radii considered is bounded above at $r_{\mathrm{pl}} \leq 1.4 r_{\oplus}$, based on evidence that suggests that planets with a radius below this are predominantly rocky. ${ }^{31}$ The lower bound on terrestrial planet radii depends on the planet's ability to retain an appreciable atmosphere, which in turn depends on their stellar illumination. This results in a dependence on the planet's semi-major axis $R_{\mathrm{pl}}$, modified by the stellar luminosity to give $r_{\mathrm{pl}} / r_{\oplus} \geq 0.8\left(R_{\mathrm{pl}} / \mathrm{AU}\right)^{1 / 2}\left(L_{\star} / L_{\odot}\right)^{-1 / 4} \cdot{ }^{32}$ These maximum and minimum radii serve as the defining limits for Earth-like planets in the simulation results presented here. The adopted ranges of planetary parameters for $\mathrm{HZ}$ planets (defined here) and gas giant planets (Sec. 5.3 below) are summarized in Table 4.

The orbital location of Earth-like planets is defined as the HZ - the region around a star where a rocky planet with a thin atmosphere may have liquid water on its surface. The location of the

Table 4 Assumed planet and dust properties.

\begin{tabular}{ll}
\hline \hline Parameter & \multicolumn{1}{c}{ Value [or (range) $]$} \\
\hline Earth-like planet geometric albedo ${ }^{\mathrm{a}}\left(A_{G}\right)$ & 0.2 \\
Earth-like planet radius $\left(r_{p}\right)$ & {$\left[0.8\left(R_{\mathrm{pl}} / \mathrm{AU}\right)^{1 / 2}\left(L_{\star} / L_{\odot}\right)^{-1 / 4}, 1.4\right] r_{\oplus}$} \\
Habitable zone $\left(R_{\mathrm{pl}}\right)$ & {$[0.95,1.67]\left(L_{\star} / L_{\odot}\right)^{1 / 2} \mathrm{AU}$} \\
Gas-giant planet geometric albedo $\left(A_{G}\right)$ & 0.3 \\
Gas-giant planet radius $^{\mathrm{b}}\left(r_{p}\right)$ & Ref. 33 $\left(r_{\text {Jup }}\right.$ max $)$ \\
${\text { Zodiacal dust brightness }\left(d F_{z} / d \Omega\right)}^{\text {Exozodi dust brightness }}{ }^{\mathrm{c}}\left(d F_{e z} / d \Omega\right)$ & Ref. 20 \\
\hline \hline
\end{tabular}

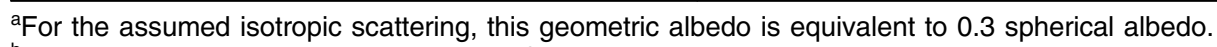

${ }^{\mathrm{b}}$ While the radius depends on the mass of the gas giant planet, we set a conservative upper limit of $r_{\text {Jup }}$.

${ }^{\mathrm{c}}$ The unit of 1 zodi is equivalent to $22 \mathrm{mag} / \mathrm{arc} \mathrm{sec}^{2}$. 
$\mathrm{HZ}$ depends both on the stellar luminosity and on assumptions for the planet's cloud properties. We adopt a conservative estimate for the $\mathrm{HZ}$ orbital radii $R_{p l}$ from 0.95 to $1.67 \mathrm{AU}$ for a Solarluminosity star. ${ }^{32,34}$ These orbital radii scale by the square root of the stellar luminosity to keep the same insolation range as the SS.

Planet sizes and semi-major axes are drawn randomly from these defined ranges for Earthlike planets, based on the distribution defined by Study Analysis Group (SAG)-13 ${ }^{35}$ and modified by Habitable exoplanet observatory (HabEx) to include the dependence of the orbital semimajor axis on the lower limit of planet radii. This is determined by drawing from the distribution defined as

$$
\frac{\partial^{2} N\left(r_{\mathrm{pl}}, P\right)}{\partial \ln r_{\mathrm{pl}} \partial \ln P}=0.38 r_{\mathrm{pl}}^{-0.19} P^{0.26}
$$

where the orbital period $P$ defines the orbital radius $R_{\mathrm{pl}}$ by way of the stellar mass $M_{\star}$ using Kepler's third law. For Earth-like exoplanets, the orbits are assumed to be circular, consistent with most previous studies, e.g. Ref. 5. The estimates made on target sensitivity take into account target availability windows (Sec. 5) determined by solar exclusions angles along with the Keplerian motion of the planet and the associated changes in planet brightness.

For the exozodiacal dust environment, we assume a constant fiducial value of 4.5 zodi based on median dust thickness, a factor derived from LBTI limits and measurements. ${ }^{11}$ Two targets of interest, epsilon Eridani and Vega, have measurements of warm dust disk brightness of 300 zodi and 33 zodi, respectively. These values are well in excess of $10 \mathrm{zodi}$, which significantly increases the integration time for detection of Earth-like planets and the risk of contamination from planet-induced disk structure. ${ }^{36,37}$ As such, these targets have been removed from the list that was presented in the Starshade Rendezvous Probe study report. ${ }^{1}$

\subsubsection{Treatment of binaries}

Nearby optical companions to potential target stars can create light leakage comparable to the starshade instrument contrast depending on their relative brightness and separation. Diffracted flux at an angle $\Theta$ away from a companion star can be approximated as $F / F_{0} \simeq 4 /\left(\pi x^{3}\right)$, where $x \equiv \pi \Theta / \lambda D$. (This formula is just the large-angle approximation for an Airy diffraction pattern; the error in this approximation is $<1 \%$ beyond the third Airy ring.) Figure 3 shows the angular separation and difference in magnitude for nearby binary stars (those with $V<5$ mag and distance $<8.5 \mathrm{pc}$ ). Diffraction from the secondary star creates additional background flux near the primary (shown for $10^{-11}, 10^{-10}$, and $10^{-9}$ contrast levels). Stars with excessive levels of background (relative to the starshade contrast floor of $4 \times 10^{-11}$ ) are dropped from the target list (open circles in Fig. 3). We note that Mu Hercules was in the target list used for the Starshade Rendezvous Probe study report ${ }^{1}$ but is removed from this updated list due to contamination from its nearby optical companion. Some wide binaries still remain as viable targets (e.g., Procyon, with a 10-magnitude-fainter white dwarf companion at 4.3" separation), shown as filled circles. While these companions are typically at 100's of AU separation, Procyon B orbits at only $15 \mathrm{AU}$ (with periapse of $9 \mathrm{AU}$ ); this relatively close orbit could impact the formation and evolution of $\mathrm{HZ}$ planets.

It is worth noting that the background contamination considered here is idealized as solely due to diffraction. Optical aberrations will contribute additional scattering. For the Roman telescope, Ref. 38 estimates that these aberrations could increase the effective contrast limit by $\sim 1$ to 2 orders of magnitude, such that borderline systems (Procyon and Sirius) would have their imaging performance significantly degraded. Very wide/faint binaries (Fomalhaut, eps Ind, bet CVn) still have insignificant contribution, even with the telescope aberrations included.

\subsubsection{Background contributions}

Each target has several sources of background noise that limit the sensitivity to Earth-like exoplanets. It is illustrative to show the relative contributions from each source as this determines which targets are viable for habitable exoplanet observations. In Fig. 4, we show the 


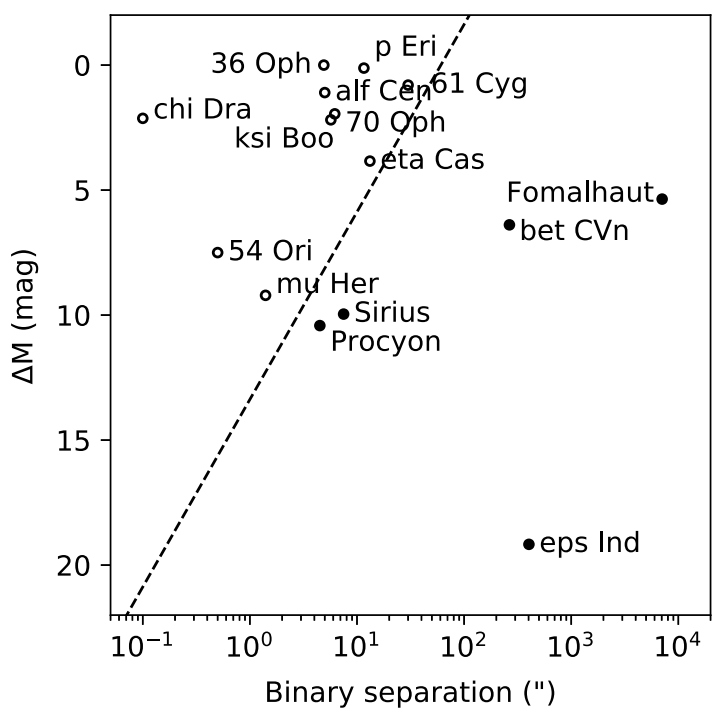

Fig. 3 Angular separation and difference in brightness are shown for all binaries that are potential targets. Diffraction from the secondary star produces a background contrast level of $4 \times 10^{-11}$ (i.e., comparable to our instrumental performance) along the dashed line. Binaries with high levels of binary contamination (i.e., those to the left of the line) are shown as open circles, while those that are still viable targets are filled circles.

contributions of photon counts, assuming 1 day of integration time in imaging mode under the following assumptions. We assume an Earth-like exoplanet at EEID in quadrature phase. The exozodiacal dust disk brightness has a fiducial value of 4.5 zodi. The leaked starlight assumes an instrument contrast $C_{S S}=4 \times 10^{-11}$ everywhere, which is conservative since the leaked starlight generally decreases away from the IWA. The SS zodi background is shown as bars indicating the range of values it can take depending on when the observation is made. Finally, the detector noise contribution is shown as a dashed line. The targets are ordered by the brightness of the Earth-like exoplanet. For most targets, the exozodiacal dust disk brightness dominates the background followed by the SS's zodiacal dust disk brightness. The leaked starlight at the IWA can be stronger in cases in which the star is very bright. It is worth noting, however, that for these stars the HZ will be pushed out to radii typically much higher than the IWA, where the leaked starlight drops. The detector noise counts lie below the contribution of SS's zodiacal dust.

The target selection is based on search completeness, discussed in more detail in the next section, which depends on the target availability windows, the FOV available around the star, and the range of parameters sampled for terrestrial exoplanets. While the photon counts estimated in Fig. 4 do not capture all of these details, they do provide a sense of which targets will provide the highest sensitivity to Earth-like exoplanets.

\subsection{Observing Windows}

The target availability windows with the Starshade/Roman system is an important constraint on the observatory's ability to spectrally characterize and determine the orbits of Earth-like exoplanets. The Starshade/Roman system can only observe stars between $54^{\circ}$ and $83^{\circ}$ from the Sun. For a star in the ecliptic plane, this limited visibility results in two observing windows per year, each $\sim 30$ days long. With increasing ecliptic latitudes, the windows become significantly longer until they merge at $54^{\circ}$ to produce a single yearly window lasting several months long and then decreasing until it vanishes above $83^{\circ}$. Stars very close to an ecliptic pole (e.g., chi Dra at $83.6^{\circ}$ ecliptic latitude) are never observable. The sky position and observing windows for all of our habitability and biosignature targets are summarized in Fig. 5, divided into the nearby-star planet search (upper panel) and the known-exoplanet sample treated in Sec. 5.2 (lower panel). The sky position and observing windows for all of our gas-giant atmospheric metallicity targets (treated in Sec. 5.3) are shown in Fig. 5. 


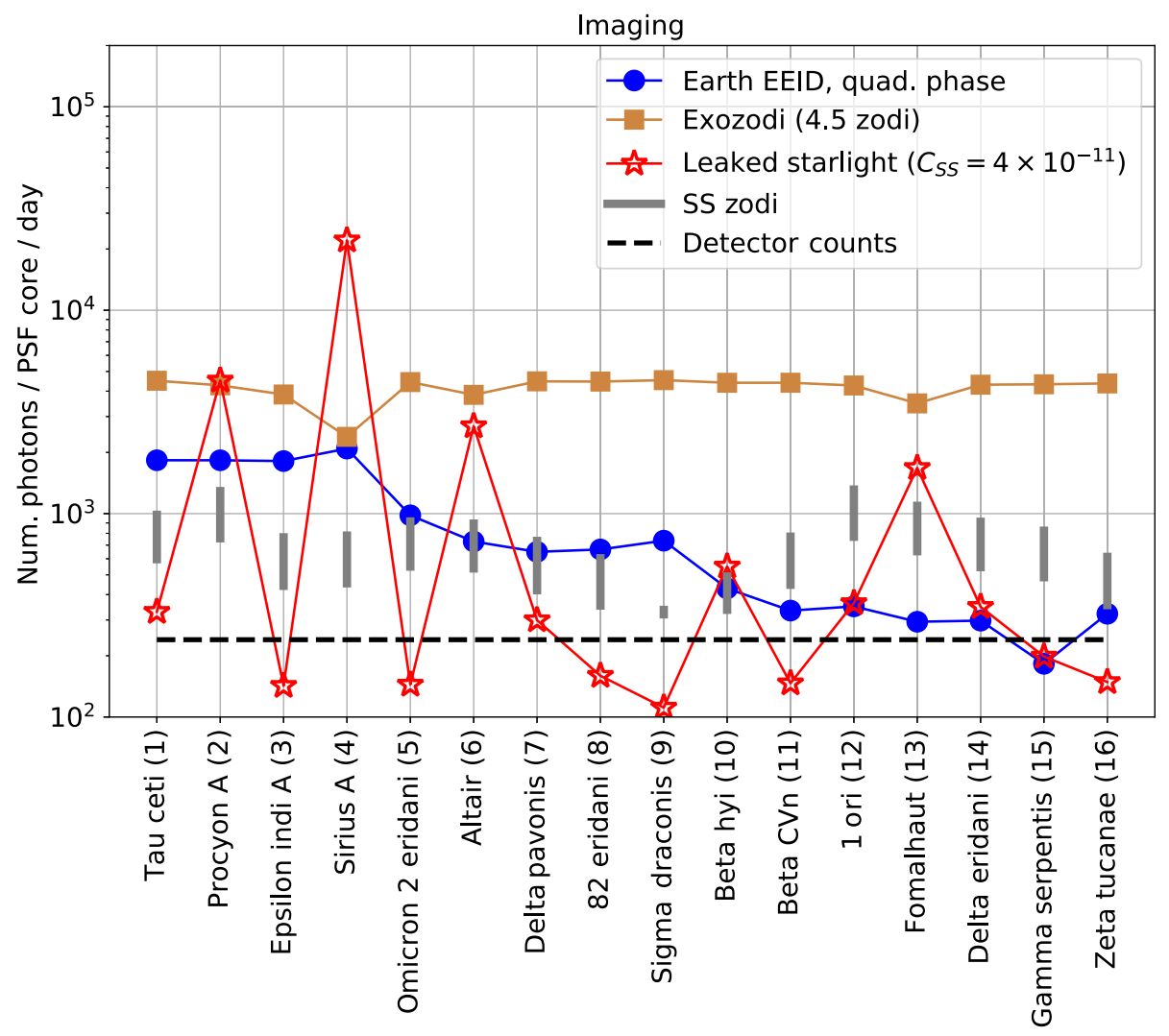

Fig. 4 Photon counts due to each contribution in the SNR model for the 16 nearby stars that provide the best sensitivity to Earth-like exoplanets in their HZs. While the Earth-like planet flux densities can vary widely depending on radius, distance to the star, and illumination phase angle, we provide estimates for a planet with Earth parameters at EEID in quadrature phase. In most cases, the exozodiacal dust disk brightness, with a fiducial brightness of 4.5 zodi, dominates the background photon contribution. The leaked starlight flux, assuming an instrument contrast $C_{S S}=4 \times 10^{-11}$, is, in most cases, comparable to the SS zodiacal (SS Zodi) dust brightness. The vertical bars of SS Zodi represent the variation depending on the time of year the target is observed. The detector noise is not a major contributor to the error budget.

The limited observing windows for each target provide a primary constraint on our observing strategy. During the two-year lifetime of the mission, there will generally be four opportunities to observe each target. While spectral characterization can be performed with only a single visit with a favorable illumination phase, multiple epochs are needed to constrain the planet's orbit, in particular its semi-major axis. The semi-major axis indicates the average amount of stellar radiation received from the parent star and thereby determines whether the planet is in the HZ.

Determining the semi-major axis with sufficient accuracy requires at least three astrometric measurements of the planet's position spread out over two years. An example of an orbit reconstruction simulation is shown in Fig. 7. Earth-like exoplanets in the HZ are generated with randomly sampled Keplerian orbital parameters with the planet's phase-varying brightness and associated astrometric precision, defined as the telescope resolution divided by imaging SNR. The simulated observations are then reconstructed with a Markov Chain Monte Carlo (MCMC) that forward models the simulated data. An ensemble of these simulations for each of our target stars demonstrates that Earth-like planets can typically be constrained to the HZ with $>80 \%$ confidence. $^{39}$

\subsubsection{Search completeness and target selection}

Our primary metric for evaluating the observatory performance for each star is target completeness - the fraction of $\mathrm{HZ}$ planets that can be completely characterized. To determine whether a 

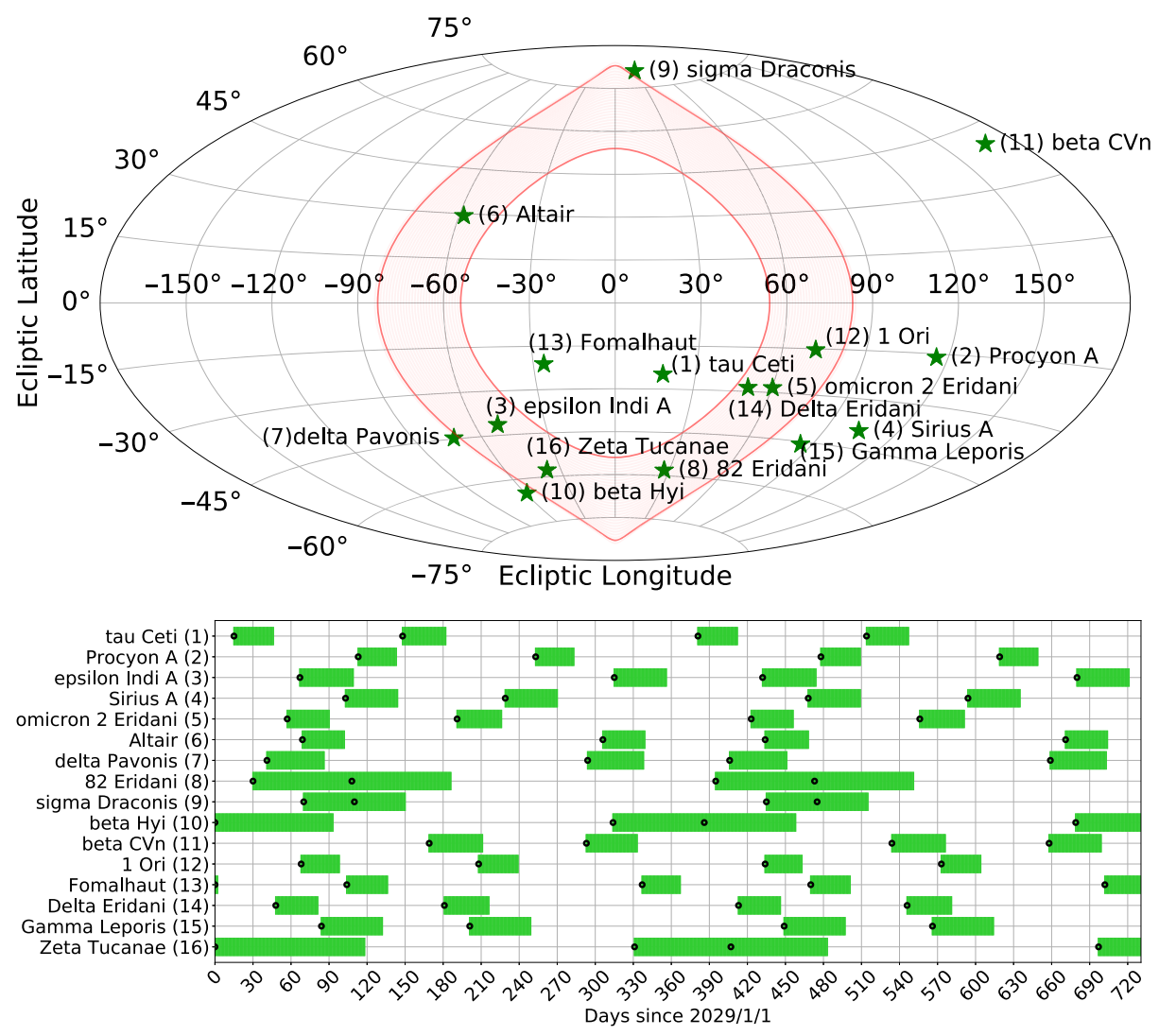

Fig. 5 Top: Sky positions of the Habitability and Biosignature Gases targets in ecliptic coordinates. An instantaneous observing region due to solar exclusion angles $\left(54^{\circ}\right.$ and $83^{\circ}$, respectively) is shown as a light red shaded region centered on $0^{\circ}$ ecliptic longitude. Bottom: Target star observing windows as constrained by telescope and starshade solar exclusion angles. These windows result from the instantaneous observing region in the panel above shifting in ecliptic longitude with a yearly period. Each star typically has two 30-day-long observing windows per year, while higher-latitude stars have a single observing window per year that is longer in duration. For the sample of nearby stars to be searched for Earth-like planets (upper panel), the black dots correspond to the desired observation start times, to allow for sufficient time for a spectral characterization.

planet is habitable, we need to be able to detect it, constrain its orbit to know that it is indeed in the $\mathrm{HZ}$, and take a spectral measurement to determine whether it has an atmosphere with biosignature gases. We, therefore, estimate the following completeness values:

1. Single-visit completeness, the fraction of $\mathrm{HZ}$ planets that can be effectively imaged at any one time (defined as SNR $>7$ within a 1-day integration) (It is worth noting that the SNR threshold was changed from 5 in the Probe Study Report1 to 7 in this study to reduce the probability of false positives. This value is consistent with the detection threshold used in the HabEx study report).

2. Orbit determination completeness, the fraction of observed planets with orbits in the HZ (assuming four observing epochs).

3. Spectral characterization completeness, fraction of imaged planets with spectra that can identify key atmospheric constituents (SNR $>20$ within a 25-day integration).

4. Target completeness, the fraction of observed planets that meet conditions 2 and 3 above.

The single visit completeness serves as a first cut to identify targets where Earth-like exoplanets have a high probability of being detected.$^{40}$ It is important to note that if a planet is not detected in a single visit, it does not mean it is absent since single visit completeness with a Starshade and CGI is $\lesssim 0.70$ (Table 5). It is equally important to note that a single detection 

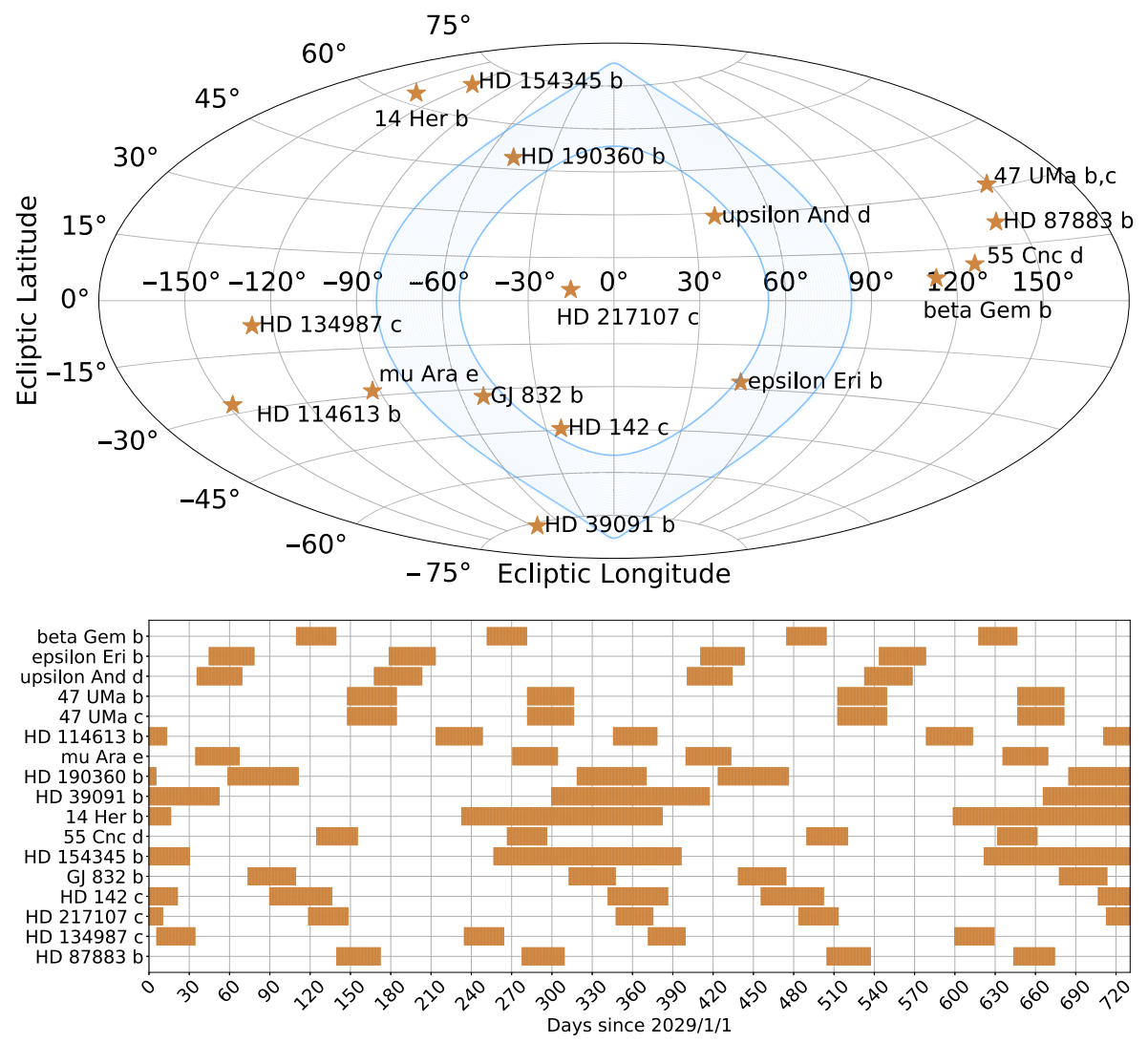

Fig. 6 Top: Sky position of known exoplanets for the gas-giant atmospheric metallicity investigation in ecliptic coordinates (Sec. 5.3). An instantaneous observing window due to solar exclusion angles $\left(54^{\circ}\right.$ and $83^{\circ}$, respectively) is shown as a light blue shaded region centered on $0^{\circ}$ ecliptic longitude. Bottom: Target star observability windows as constrained by telescope and starshade solar exclusion angles.

of a planet in a region consistent with the habitable is not enough to conclude that it is indeed a HZ exoplanet. Follow-up observations that constrain the planet's orbits are necessary to determine that.

The orbit determination completeness is the probability that the planet's orbit can be constrained to be in the HZ. In a separate study, ${ }^{39}$ it was determined that three detections in four visits to the target were sufficient to constrain the orbit of a $\mathrm{HZ}$ exoplanet with $>80 \%$ confidence, depending on the orbital inclination and the phase of observation. The orbit determination completeness, in this study, is the probability that at least three detections occur with four visits. The simulations in that study sample the orbit periods and observation windows assumed here and perform a MCMC fit of the observations to estimate the posterior distribution of the planet's semi-major axis. The number of visits is limited by the lengths and periodicity of the target availability windows for most stars of interest (see Fig. 5).

The spectral characterization completeness is the probability that a spectroscopic observation of a target is successful in any one of four visits. The criteria of success (SNR $>20$ ) is based on a study by Ref. 41, which found that this is was the minimum needed for detection of molecular oxygen and water vapor lines in the CGI band. The 25-day integration time window is the typical maximum for most targets, although some are available for significantly longer.

The target completeness requires all criteria above are met; it is the probability that the orbit constraint requirements and a spectral observation is achieved for a nearby star. For each system, we calculate the completeness with a Monte Carlo sampling of $\mathrm{HZ}$ orbits. We sample random semi-major axes [using Eq. (10)], orbital inclinations (cosine distributed), and true anomalies (uniformly distributed for a circular orbit). Circular orbits are assumed. Each randomly selected planet is propagated along its Keplerian orbit, with the time of observation limited to four 

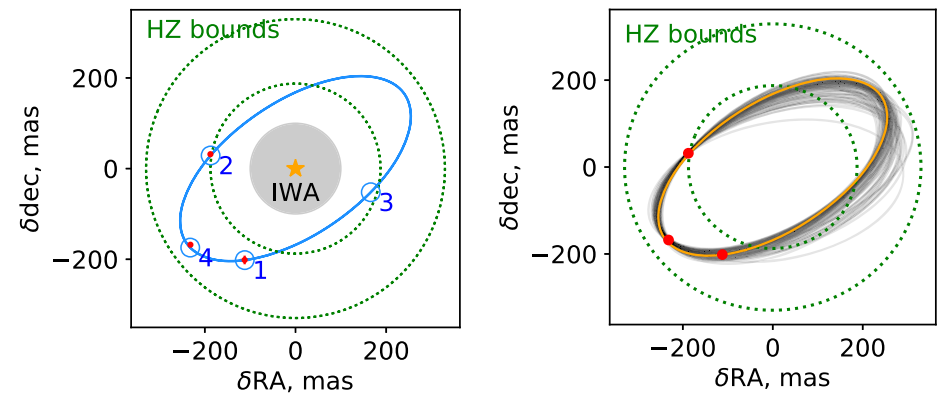

Fig. 7 Four visits of tau Ceti, as planned with Starshade Rendezvous Probe (Fig. 5), with three detections are sufficient to constrain orbits to the $\mathrm{HZ}$ (shown in dashed green lines). The left panel shows a simulation of an Earth-like planet with a circular orbit. True positions marked in blue circles and astrometric estimates with error bars shown in red. The numbers indicate the visit number for each observation. The IWA is shown in gray. On the right, sample orbits (gray lines) from a MCMC-reconstructed posterior distribution demonstrate that the fit is well within the $\mathrm{HZ}$, including uncertainties in the orbit eccentricity. The true orbit is shown in orange.

Table 5 Nearby stars targeted for Earth-like planets

\begin{tabular}{|c|c|c|c|c|c|c|c|c|c|c|}
\hline \multirow[b]{2}{*}{ Star name } & \multirow[b]{2}{*}{$\begin{array}{l}\text { Distance } \\
\text { (pc) }\end{array}$} & \multirow[b]{2}{*}{$V$ (mag) } & \multirow[b]{2}{*}{$L_{\star}\left(L_{\odot}\right)$} & \multirow[b]{2}{*}{$T_{\text {eff }}(\mathrm{K})$} & \multirow[b]{2}{*}{$M_{\star}\left(M_{\odot}\right)$} & \multirow[b]{2}{*}{$\begin{array}{l}\text { Spectral } \\
\text { type }\end{array}$} & \multicolumn{4}{|c|}{ Completeness } \\
\hline & & & & & & & $\begin{array}{l}\text { Single- } \\
\text { visit }\end{array}$ & Orbit & Spectral & Overall \\
\hline tau Ceti ${ }^{a, b}$ & 3.7 & 3.5 & 0.52 & 5283 & 0.80 & G8.5V & 0.67 & 0.55 & 0.79 & 0.48 \\
\hline Procyon $^{c}$ & 3.5 & 0.4 & 7.1 & 6543 & 1.49 & F5IV-V & 0.65 & 0.54 & 0.55 & 0.43 \\
\hline eps Ind ${ }^{b, c}$ & 3.6 & 4.7 & 0.23 & 4683 & 0.68 & $\mathrm{~K} 4 \mathrm{~V}$ & 0.67 & 0.52 & 0.74 & 0.42 \\
\hline Sirius $^{c}$ & 2.6 & -1.4 & 30.5 & 9580 & 2.40 & A1.0V & 0.58 & 0.52 & 0.25 & 0.25 \\
\hline omi $2 \mathrm{Eri}^{\mathrm{b}}$ & 5.0 & 4.4 & 0.42 & 5151 & 0.81 & $\mathrm{~K} 0.5 \mathrm{~V}$ & 0.65 & 0.51 & 0.21 & 0.13 \\
\hline Altair & 5.1 & 0.8 & 10.7 & 7800 & 1.83 & A7IV-V & 0.58 & 0.52 & 0.10 & 0.09 \\
\hline del Pav & 6.1 & 3.5 & 1.3 & 5590 & 0.99 & G8.0IV & 0.64 & 0.55 & 0.07 & 0.05 \\
\hline $\mathrm{Eri}^{\mathrm{b}}$ & 6.0 & 4.3 & 0.69 & 5401 & 0.85 & G8.0V & 0.60 & 0.38 & 0.02 & 0.01 \\
\hline sig Dra & 5.8 & 4.7 & 0.44 & 5246 & 0.80 & G9.0V & 0.55 & 0.39 & 0.00 & 0.00 \\
\hline bet Hyi & 7.5 & 2.8 & 3.7 & 5873 & 1.14 & G1IV & 0.58 & 0.51 & 0.00 & 0.00 \\
\hline bet $C V n^{c}$ & 8.4 & 4.2 & 1.3 & 5930 & 1.03 & GOV & 0.43 & 0.13 & 0.00 & 0.00 \\
\hline Ori & 8.1 & 3.2 & 3.0 & 6424 & 1.24 & F6V & 0.50 & 0.30 & 0.00 & 0.00 \\
\hline Fomalhaut ${ }^{\mathrm{a}, \mathrm{c}}$ & 7.7 & 1.2 & 16.5 & 8399 & 2.05 & A3V & 0.46 & 0.43 & 0.00 & 0.00 \\
\hline del Eri & 9.0 & 3.5 & 3.4 & 5095 & 1.19 & KOIV & 0.46 & 0.25 & 0.00 & 0.00 \\
\hline gam Lep & 8.9 & 3.6 & 2.5 & 6372 & 1.27 & F7V & 0.44 & 0.21 & 0.00 & 0.00 \\
\hline zet Tuc & 8.6 & 4.2 & 1.3 & 5948 & 1.01 & GOV & 0.42 & 0.14 & 0.00 & 0.00 \\
\hline
\end{tabular}

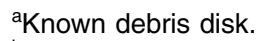

${ }^{\mathrm{b}}$ Known to have planet(s).

'Binary (see Fig. 3). 


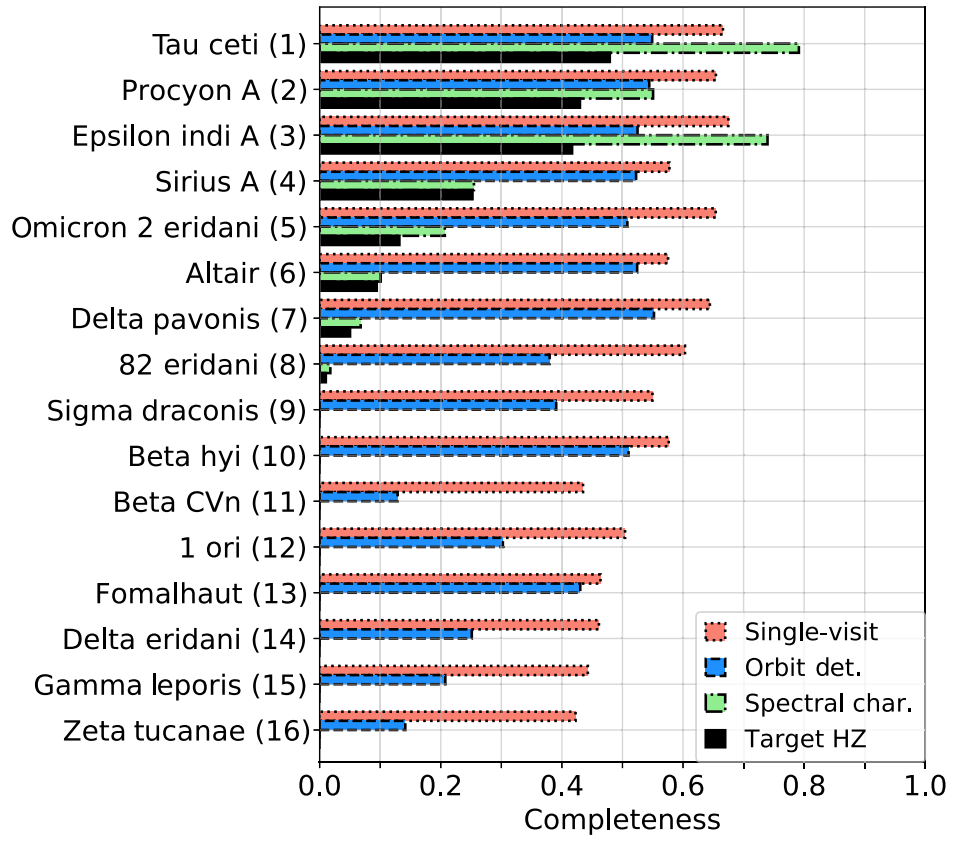

Fig. 8 Completeness estimates are shown for the detection of Earth-like planets around nearby stars. The simulation results are sorted from best target completeness (top) to least (bottom). The overall target completeness is shown in black, while its composite factors are the single-visit completeness (orange), orbit determination completeness (blue), and spectral characterization completeness (green).

observing windows spaced over two years (see Fig. 5 in Sec. 5.2). A list of 16 stars for finding Earth-like planets are listed in Table 3 and summarized in Fig. 8, which shows simulation results for single visit, orbit determination, spectral characterization, and the overall target completeness for each target.

Most of the $\mathrm{HZ}$ is visible for all of the targets, with single-visit completeness ranging from $\sim 0.5$ to $\sim 0.7$ (red bars in Fig. 8). Most of the planets that are visible in their HZ can also have their orbits traced over multiple epochs, resulting in orbit determination completenesses of $\gtrsim 0.5$ for most stars (blue in Fig. 8). The ability of the observations to constrain each planet's orbit will be described in a companion paper. ${ }^{39}$

The predominant limiting factor is the spectral characterization completeness (green in Fig. 8), which varies by orders of magnitude between stars. Only the brightest stars provide enough photons to produce a high-quality reflected light spectrum within the integration time limits. For the best targets, spectral characterization completeness can be as high as $\sim 0.8$, but as the expected planetary reflected light flux density decreases, 25 days is not sufficient to achieve a spectral SNR $>20$. Although these fainter planets will not meet our primary science objective, the probability of detection and orbit constraint is still very high, and lower-SNR spectra will still be sensitive to some atmosphere types. This will be the subject of a future investigation.

Table 6 Known super-Earth exoplanet target list.

\begin{tabular}{|c|c|c|c|c|c|c|c|c|c|c|c|c|c|}
\hline \multirow{2}{*}{$\begin{array}{l}\text { Planet } \\
\text { name }\end{array}$} & \multirow{2}{*}{$\begin{array}{l}\text { Distance } \\
\quad(p c)\end{array}$} & \multirow{2}{*}{$\begin{array}{c}V \\
\text { (mag) }\end{array}$} & \multirow{2}{*}{$\begin{array}{c}L_{\star} \\
\left(L_{\odot}\right)\end{array}$} & \multirow{2}{*}{$\begin{array}{l}T_{\text {eff }} \\
(\mathrm{K})\end{array}$} & \multirow{2}{*}{$\begin{array}{c}M_{\star} \\
\left(M_{\odot}\right)\end{array}$} & \multirow{2}{*}{$\begin{array}{c}\text { Spectral } \\
\text { type }\end{array}$} & \multirow{2}{*}{$\begin{array}{c}M_{p} \\
\left(M_{\oplus}\right)\end{array}$} & \multicolumn{2}{|c|}{$a_{p}$} & \multirow[t]{2}{*}{$F_{p} / F_{\star}$} & \multicolumn{3}{|c|}{ a Integration time (days) ${ }^{b}$} \\
\hline & & & & & & & & $A U$ & (mas) & & $\beta=45^{\circ}$ & $\beta=90^{\circ}$ & $\beta=135^{\circ}$ \\
\hline tau Ceti e & 3.7 & 3.49 & 0.5 & 5283 & 0.8 & G8.5V & 3.9 & 0.54 & 147 & 1.86 & 0.14 & 0.44 & 9.3 \\
\hline tau Ceti $f$ & 3.7 & 3.49 & 0.5 & 5283 & 0.8 & G8.5V & 3.9 & 1.33 & 365 & 0.30 & 1.9 & 8.4 & - \\
\hline
\end{tabular}

Note. - Dashes indicate integration times in excess of 25 days.

aPlanet-star flux ratio for a half-illuminated planet $\left(\beta=90^{\circ}\right)$.

${ }^{b}$ Integration times to reach $\mathrm{SNR}=20$ for an $R=50$ spectra, over a range of illumination phase angles $\beta$. 
Table 7 Known gas-giant exoplanet target list.

\begin{tabular}{|c|c|c|c|c|c|c|c|c|c|c|c|c|c|}
\hline \multirow{2}{*}{$\begin{array}{l}\text { Planet } \\
\text { Name }\end{array}$} & \multirow{2}{*}{$\frac{\text { distance }}{(\mathrm{pc})}$} & \multirow{2}{*}{$\frac{V}{(\mathrm{mag})}$} & \multirow{2}{*}{$\frac{L_{\star}}{\left(L_{\odot}\right)}$} & \multirow{2}{*}{$\frac{T_{\text {eff }}}{(\mathrm{K})}$} & \multirow{2}{*}{$\frac{M_{\star}}{\left(M_{\odot}\right)}$} & \multirow{2}{*}{$\frac{\text { Spectral }}{\text { Type }}$} & \multirow{2}{*}{$\frac{M_{p}}{\left(M_{\text {Jup }}\right)}$} & \multicolumn{2}{|c|}{$a_{p}$} & \multirow{2}{*}{$\frac{F_{p} / F_{\star}{ }^{a}}{\left(\times 10^{-9}\right)}$} & \multicolumn{3}{|c|}{$\begin{array}{l}\text { Integration time } \\
\text { (days) }^{\mathrm{b}}\end{array}$} \\
\hline & & & & & & & & $\mathrm{AU}$ & (mas) & & $\beta=45^{\circ}$ & $90^{\circ}$ & $135^{\circ}$ \\
\hline bet Gem b & 10.4 & 1.16 & 40.9 & 4850 & 2.6 & Kolllvar & 2.30 & 1.64 & 158 & 10.9 & $\leq 0.1$ & $\leq 0.1$ & $\leq 0.1$ \\
\hline gam Cep bc & 14.1 & 3.21 & 11.8 & 4761 & 1.9 & K1IV & 1.85 & 2.05 & 145 & 7.13 & $\leq 0.1$ & $\leq 0.1$ & 0.5 \\
\hline Ups and d & 13.5 & 4.09 & 3.6 & 6213 & 1.3 & F8V & 4.13 & 2.51 & 186 & 4.43 & $\leq 0.1$ & 0.3 & 7 \\
\hline eps Eri b & 3.2 & 3.71 & 0.4 & 5146 & 0.9 & $\mathrm{~K} 2.0 \mathrm{~V}$ & 1.55 & 3.39 & 1055 & 2.65 & $\leq 0.1$ & 0.3 & 7 \\
\hline UMa b & 14.1 & 5.03 & 1.7 & 5882 & 1.1 & GOV & 2.53 & 2.10 & 149 & 6.57 & $\leq 0.1$ & 0.6 & 16 \\
\hline UMa c & 14.1 & 5.03 & 1.7 & 5882 & 1.1 & GOV & 0.54 & 3.60 & 255 & 2.59 & 0.8 & 3.5 & - \\
\hline HD $192310 c^{c}$ & 8.9 & 5.72 & 0.4 & 5080 & 0.8 & K3V & 0.08 & 1.18 & 132 & 3.29 & 0.6 & 2 & - \\
\hline HD $219134 h^{c}$ & 6.5 & 5.57 & 0.3 & 4835 & 0.8 & $\mathrm{~K} 3.0 \mathrm{~V}$ & 0.34 & 3.11 & 475 & 2.79 & 0.7 & 3 & - \\
\hline HD 39091b & 18.3 & 5.65 & 1.6 & 5950 & 1.1 & G1V & 10.02 & 3.10 & 169 & 2.67 & 1 & 6 & - \\
\hline HD 114613b & 20.7 & 4.84 & 4.5 & 5782 & 1.2 & G3V & 0.36 & 5.34 & 258 & 1.00 & 2 & 10 & - \\
\hline HD 190360b & 15.9 & 5.73 & 1.2 & 5552 & 1.0 & G6IV & 1.54 & 3.97 & 250 & 1.93 & 3 & 15 & - \\
\hline HD $160691 \mathrm{c}$ & 15.5 & 5.12 & 2.0 & 5784 & 1.1 & G3IV/V & 1.81 & 5.24 & 337 & 1.09 & 3 & 15 & - \\
\hline Her b & 17.6 & 6.61 & 0.7 & 5388 & 1.1 & KOV & 4.66 & 2.93 & 166 & 3.20 & 4 & 19 & - \\
\hline Cnc d & 12.3 & 5.96 & 0.7 & 5235 & 1.0 & G8V & 3.88 & 5.50 & 445 & 0.92 & 11 & - & - \\
\hline HD 154345 b & 18.6 & 6.76 & 0.7 & 5468 & 0.9 & G8V & 0.82 & 4.21 & 226 & 1.80 & 21 & - & - \\
\hline HD 217107 c & 19.9 & 6.16 & 1.2 & 5704 & 1.1 & G8IV/V & 2.60 & 5.32 & 267 & 1.02 & 20 & - & - \\
\hline HD 142 c & 25.7 & 5.70 & 3.0 & 6249 & 1.2 & F7V & 5.30 & 6.80 & 264 & 0.58 & 16 & - & - \\
\hline eps Ind $A b^{c}$ & 3.6 & 4.69 & 0.2 & 4683 & 0.7 & $\mathrm{~K} 4 \mathrm{~V}$ & 3.25 & 11.55 & 3188 & 0.21 & 14 & - & - \\
\hline GJ $229 b^{c}$ & 5.8 & 8.15 & 0.1 & 3709 & 0.5 & M1.5V & 0.03 & 0.90 & 156 & 1.66 & 12 & - & - \\
\hline GJ 832 b & 4.9 & 8.67 & 0.0 & 3601 & 0.4 & M1.5V & 0.68 & 3.56 & 719 & 2.56 & 24 & - & - \\
\hline
\end{tabular}

Note. - Dashes indicate integration times in excess of 25 days.

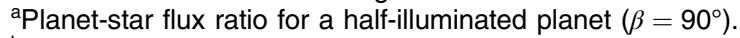

bintegration times to reach SNR $=15$ for an $R=50$ spectra, over a range of illumination phase angles $\beta$.

'Planet not listed in the SRP study report. ${ }^{1}$

Of the total of eight stars that have non-zero target completeness, tau Ceti has the largest $(\gtrsim 0.5)$. The remaining targets still have a significant completeness for detection and orbit determination of Earth-like exoplanets. These stars are of interest for reconnaissance of planets in orbit and for observing their exozodiacal dust disk brightness in preparation for more sensitive observatories in the future.

It is worth noting that tau Ceti has two already-discovered super-Earth planets that are widely separated and bright enough to have their atmospheres characterized. ${ }^{42}$ Integration times to obtain spectra are given in Table 6 as a function of each planet's illumination phase.

Other than the observing window limitations on integration time, the analysis presented here is on a per-target basis and has not assumed any constraints on retargeting time or total mission duration. Nevertheless, there is a effective upper bound on number of targets set by our requirements (orbit determination and spectral characterization). While the number of target stars could be increased with a greater allocation of telescope time, the integration time needed to achieve a 
successful spectral measurement is limited by the solar exclusion angles. The Roman Space Telescope CGI with starshade is therefore more limited by sensitivity than it is by telescope time allocation.

\subsection{Sensitivity to Gas-Giant Planets}

Next, we consider known gas-giant planets as targets for atmospheric characterization. The goal here is to determine whether there is a correlation between atmospheric metallicity and fundamental planetary properties such as mass and semi-major axis (Fig. 9). A strong correlation is found in SS gas giants, and there have been indications of such a correlation in exoplanet data, although the uncertainties in the atmospheric metallicity of exoplanets are still high (Fig. 9). Measurements of the methane absorption line serve as our primary proxy for the atmospheric metallicity, allowing for direct comparison with the SS's outer planets. ${ }^{43}$ Our quantitative objective is to achieve a measurement in the correlation between planet mass and atmospheric metallicity with at least $3-\sigma$ significance.

To determine if such a correlation is present in a population of gas giant exoplanets orbiting different stars, we need to establish how many are needed and with what level of uncertainty in metallicity. There are currently 20 gas giant exoplanets with orbital angular separations accessible to the Starshade Rendezvous Probe (from 0."13 to 3." 2 ; Table 7). Since it is unrealistic to expect all of them will be at an orbital phase favorable for spectral measurements, we looked at randomly sampled subsets that might be available. We find that if a subset of 10 stars is available with a $30 \%$ metallicity fraction uncertainty, then it is possible to discern a mass-metallicity correlation with $3-\sigma$ statistical significance (Fig. 10). The 30\% metallicity fractional uncertainty can be achieved with spectral SNR $>15$ measurements in one or two bands from $~ 600$ to $\sim 800 \mathrm{~nm}^{.44,45}$

The mission requirements based on the driving investigation (detecting Earth-like planets) enable measurement of known giant-planet metallicities. Integration times depend on the illumination phase angle during observation; while the orbital phase for radial-velocity-detected planets is known, the orbital inclination is uncertain. Table 7 shows the integration times required to achieve spectral SNR $>15$ for a range of illumination phase angles. A geometric albedo of 0.3 is assumed, with radius constrained by the mass-size relation of Ref. 33 but conservatively capped at 1 Jupiter radius. This albedo is a conservative choice, well below Jupiter's actual value of $0.5^{15,46}$ but consistent with models of Jupiter-mass planets located closer to the Sun. ${ }^{15,44}$ It is worth noting that the expected integration times have changed somewhat from what was reported in the probe study report ${ }^{1}$ due to changing the geometric albedo from 0.5 to 0.3 and the shift from

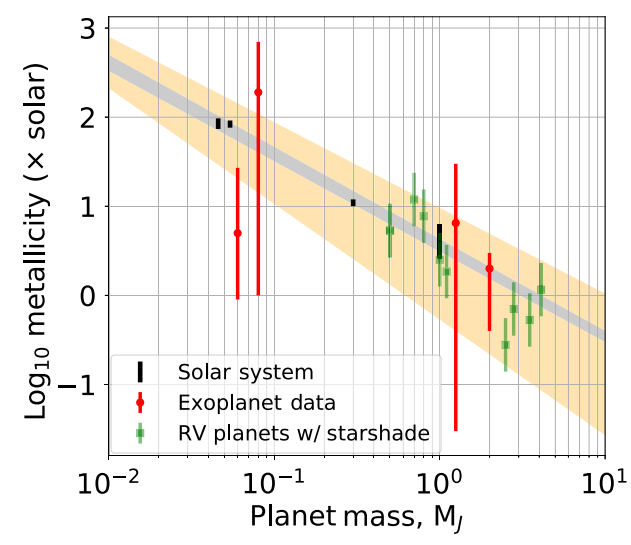

Fig. 9 The correlation of atmospheric metallicity and planet mass. The data, with uncertainties, is shown for the SS (black bars) and exoplanet transit spectroscopy measurements ${ }^{12}$ (red bars). The green points show a random sample of 10 known gas giant exoplanets that could be observed with the Starshade Rendezvous Probe assuming they follow the same correlation with $30 \%$ fractional uncertainty in atmospheric metallicity. 


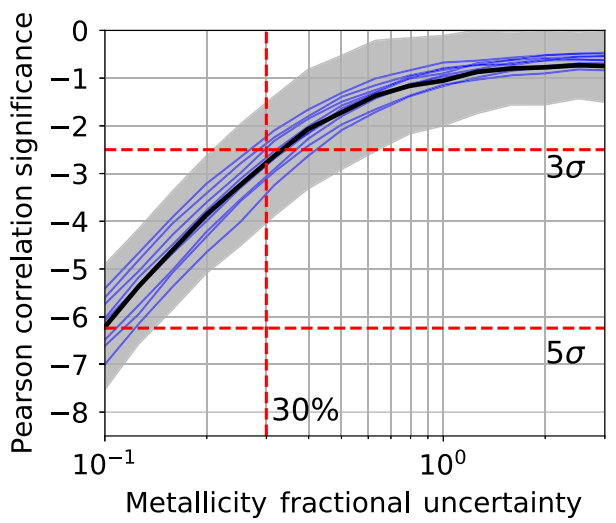

Fig. 10 Our ability to fit the mass-metallicity relationship among known gas-giant planets depends on the accuracy of the individual metallicity measurements. For several sets of 10 randomselected targets, the statistical significance of the Pearson correlation coefficient is estimated over a range of metallicity fractional uncertainties (purple lines). The median result is in black, with a gray band as the full range of uncertainties in the Pearson correlation significance. We find that a $30 \%$ metallicity uncertainty is sufficient to assess the mass-metallicity relationship. The gray band shows the uncertainty on the Pearson correlation significance corresponding to the single instance represented by the thick black line.

the integral field spectrometer to the slit prism spectrometer, which changed the current best estimate of the end-to-end efficiency from $2.5 \%$ to $3.4 \%$.

Integration times are on the order of several days for the majority of targets at favorable illumination angles. Ten spectra can be obtained with a total of 50 days of integration time allocated among the most favorable targets. It is worth noting that, by the time starshade begins operations, this target list is expected to have grown, providing even more flexibility in scheduling. The observing windows for current targets are plotted in Fig. 6, which shows whether a target is available for observation at any given time during operations. Roman-CGI observations, continued Doppler monitoring, and Gaia astrometry will constrain the brightness and orbital parameters prior to starshade operations, so these observations can be precisely planned for maximum planet visibility with no need for revisits.

\subsection{Sensitivity to Exozodiacal Dust}

The dust surrounding Earth-like planets is small in mass but large in area, making it generally much easier to observe than the planet itself. The integrated flux from SS's zodiacal dust, for example, is orders of magnitude brighter than the Earth. Imaging the dust disk distribution requires an integration time of 1 day (on average) or up to 4 days (maximum) to obtain a flux sensitivity of 0.1 zodi, enabling $5-\sigma$ detection of disks as faint as 0.5 zodi. While disks this faint have never been observed (other than the SS, with 1 zodi), the median level inferred from a sample of nearby stars is 4.5 zodis, ${ }^{11}$ suggesting that most, if not all, of the systems with $\mathrm{HZ}$ dust will be detected. With a telescope imaging resolution of $0 .{ }^{\prime \prime} 065$, the disks will be mapped at spatial resolutions of $\sim 0.2$ to $0.5 \mathrm{AU}$.

With this sensitivity, it may be possible to detect the influence of planets on the zodiacal dust disk structure. 33 have shown that planets with $\gtrsim 4$ Earth masses can introduce significant features on the dusk disk brightness distribution of moderately bright dusts disks ( $\sim 6$ to 10 zodis). The induced structure would be located in close proximity to the observed planet with both following the same orbital trajectory, removing any ambiguity in whether the disk structure is planet related.

\section{Observing Strategy}

The science objectives are met with an observing program that balances detection and characterization of new exoplanets with the characterization of known giant planets. The observing 
strategy is guided by the sensitivity toward individual targets (Sec. 5) and fundamental limits on the targets' visibility.

\subsection{Earth-Like Planets}

Having already identified the best targets for detection of Earth-like planets for SRP given its constraints (Sec. 5.1), we now describe our strategy on how to schedule observations to optimize the number of characterized planets. The eight most promising stars will be given priority for at least one visit. The revisit strategy for these targets depends on the information gathered during each visit.

The decision tree used for observations is shown in Fig. 11 and described in detail here. The decision tree assumes that the imaging observation is complete with $\sim 1$ day of integration and the data are expected to be available for analysis within a couple of days. In the time between the observation and data retrieval, the Roman telescope will be available for other observations while the starshade remains in position. The starshade science team will have fast analysis tools in hand to estimate the brightness of the exozodiacal dust disk and detect Earth-like exoplanet candidates. Based on the findings, the starshade team can either decide to initiate the cruise into position for observing the next target in the sequence or to initiate a long integration time observation for spectral characterization.

The first visit is a reconnaissance observation. The first check is whether the system has a exozodiacal dust disk above or below 10 zodi. While some of our target stars have existing upper limits on their exozodiacal dust disk brightness, based on precision nulling measurements of their dust's infrared emission, they are not sufficient to rule out deleterious levels of dust. It is worth noting that, while we have used a fiducial value of 4.5 zodi in Secs. 5 and 7 , for the purposes of estimating background, in reality the exozodiacal dust brightness will vary from target to target, and we assume it will be unknown prior to the first observation. Here, we are describing the tentative design reference mission for the Starshade Rendezvous Probe mission. If the disk is brighter than 10 zodi, the target is removed from the habitability and biosignatures target list since it is not expected that an Earth-like exoplanet could be spectrally characterized

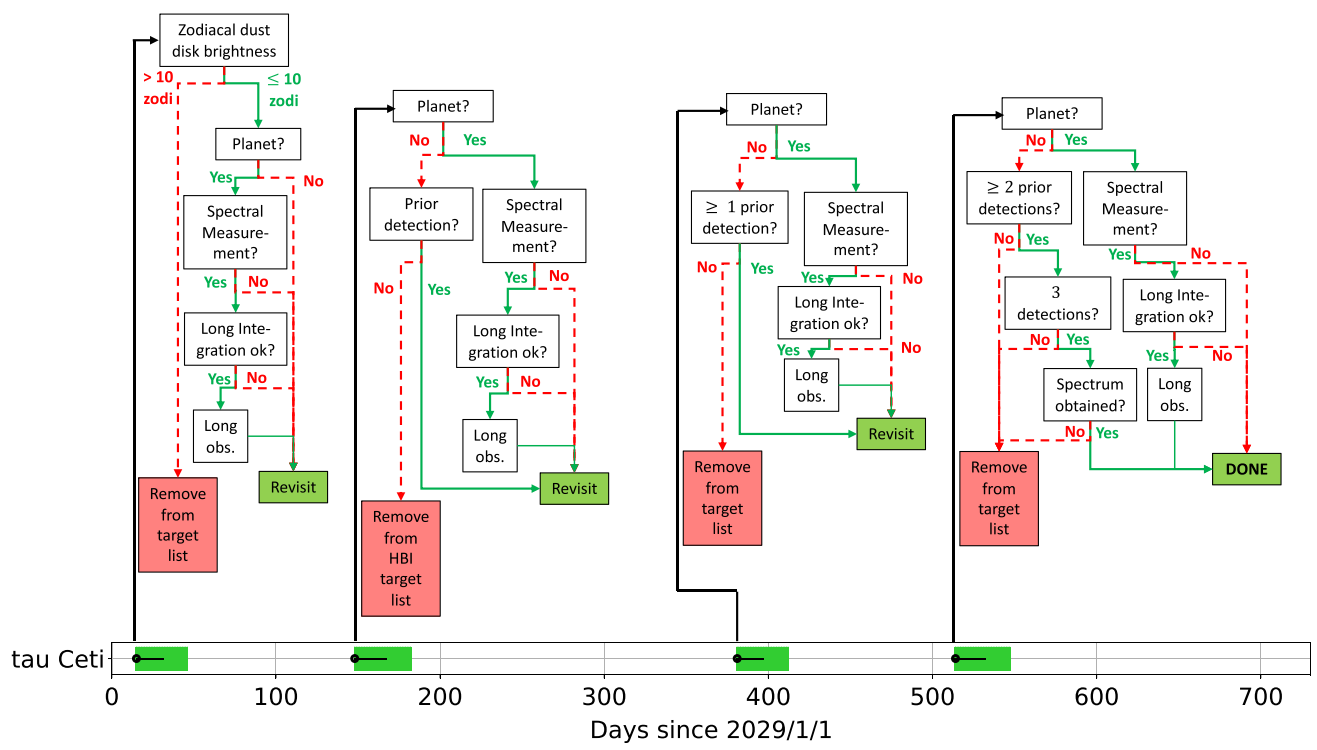

Fig. 11 The decision tree for discovery of Earth-like planets adapts its observations as new information is gathered. Depending on (1) the amount of exozodiacal dust, (2) the number of observations of a planet candidate, and (3) whether long spectroscopic integration times are executed, a target may either be revisited or removed from the target list. The two-year timeline on the bottom of the figure shows the windows available for observations (shown here with tau Ceti's observing windows). 
against such a bright background, and there is an increased risk of false-positive planet detections from planet-induced dust structures. ${ }^{37}$ If a target is removed, the observation plan will be updated with the next best target, which may or may not be visited at a later period depending on what is discovered for the target ensemble.

If the analysis finds that the exozodiacal dust disk is $\leq 10$ zodi but no planet consistent with $\mathrm{HZ}$ orbit is found, the target is kept on the list for a revisit as a planet could still appear in subsequent observations. If a planet consistent with an $\mathrm{HZ}$ orbit is found, then the imaging data will provide the brightness of the planet, which determines whether a spectral measurement with SNR $>20$ is achievable in the remainder of the observing time window, typically 25 days. If that is the case, a spectral observation will be initiated.

On the second visit to a target, if no planet consistent with an $\mathrm{HZ}$ orbit has been detected, then the target is removed and the observation plan is updated with the next best target in the list. If the planet is detected either for the first or second time, a decision is made based on the data to take a spectroscopic measurement as described above. Spectroscopic measurements are only required to be performed once, so if such a measurement was made in the first visit, then it will not be repeated.

On the third visit, if the planet consistent with an $\mathrm{HZ}$ orbit has only been detected once, no further visits will be planned since at least three observations in four planned visits are required. However, there is some flexibility in this decision since the third visit will be made in the second year of observations and the science team will have additional information on the exozodiacal dust disk brightness and population of planet candidates in the ensemble of targets already observed. In the event that there are a small number of target systems left, then this system could have more visits planned. If there are a large number of relevant target systems still available, this revisit priority may fall lower than the priority of these other systems, recognizing that revisit priority may evolve as knowledge is gained about each system. Spectroscopic measurements can be triggered based on the criteria discussed above.

In the event that the planet has only been observed twice before and/or no spectroscopic measurement has been made yet, a fourth visit will be needed to determining whether the planet's orbit is in the HZ. This will occur during the last $\sim 6$ months of the mission, so the prioritization of targets could be significantly affected by how many Earth-like exoplanet candidates have been found and their potential for a spectral measurements with SNR $\geq 20$.

\subsection{Known Gas-Giant Planets}

Unlike the habitability and biosignatures targets, it is expected that significantly more information about the known gas giants will be available. Although we have not assumed prior information on the targets obtained with the CGI in this study, it is possible that Roman-CGI will have already observed these systems and directly imaged the planets before Starshade operations begin. This will determine how bright they are and whether or not spectral measurements with SNR $\geq 15$ are viable. The top-ranked targets with their estimated integration time will be integrated into the observation plan.

\subsection{Exozodiacal Dust}

No additional observations are required to observe the dusty debris that is prevalent in planetary systems; it will be detected alongside any observed planets.

For the aim of characterizing the influence of planets in the dust disk distribution, target stars with bright exozodi ( 6 to 10 zodi) that show clumps with $\geq 10 \%$ excess brightness may be revisited up to three more times. Identifying Earth-like planets takes priority, but in the event that exozodiacal dust is too bright in most stars and systems with the characteristics described above exist, these observations will be executed. These observations will track the motion of dust disk clumps to test whether their orbits are Keplerian, indicative of an associated planet. Provided a system within this exozodi range with a $\geq 4 M_{\oplus}$ planet is found, this measurement will provide a means to probe planetary systems in stars with high levels of exozodiacal dust in their HZs. ${ }^{36}$ 


\subsection{Scheduling}

The visit strategy needs to be dynamic since it will be modified with each observation of habitability targets while at the same time ensuring that a subset of 10 known gas giants can be spectrally characterized with retargets that optimize the fuel usage. Since there is a significant amount of uncertainty associated with the distribution of exozodiacal dust disks and the frequency of occurrence of Earth-like exoplanets, this requires a fairly sophisticated Monte Carlo simulation that demonstrates that the decision tree is adaptable to the full range of possibilities. This will be the subject of a future study.

In the Starshade Probe Study report, the delta-v allocated to retargeting was $1100 \mathrm{~m} / \mathrm{s} .{ }^{1}$ An additional $300 \mathrm{~m} / \mathrm{s}$ of delta- $\mathrm{v}$ is allocated for stationkeeping. The allocation for large slews was estimated to be sufficient for 36 retargeting maneuvers using a limiting scenario in which nine targets were visited four times each (Fig. 12). For retargeting within other starshade mission concepts, see Refs. 5 and 8. While our objective is to visit 10 habitability and biosignatures targets, some with multiple revisits, and 10 known gas giants only once, the extreme scenario bounds the fuel usage because it assumes the need to retarget in situations that are not necessarily the most fuel efficient. The gas giants, for example, can be visited when they are close to the path needed for other targets, resulting in significantly smaller fuel burns.

The main driver for delta- $\mathrm{v}$ is the need to visit targets 4 times in a period of two years. If the mission duration could be made longer, the retargeting strategy could follow the natural right ascension progression of observing windows as the Earth rotates around the Sun, which can

\begin{tabular}{|lllllllllllllllllllllllll|l|}
\hline \multicolumn{1}{|c|}{2029} & \multicolumn{7}{c|}{2030} & & $2031+$ \\
\hline
\end{tabular}
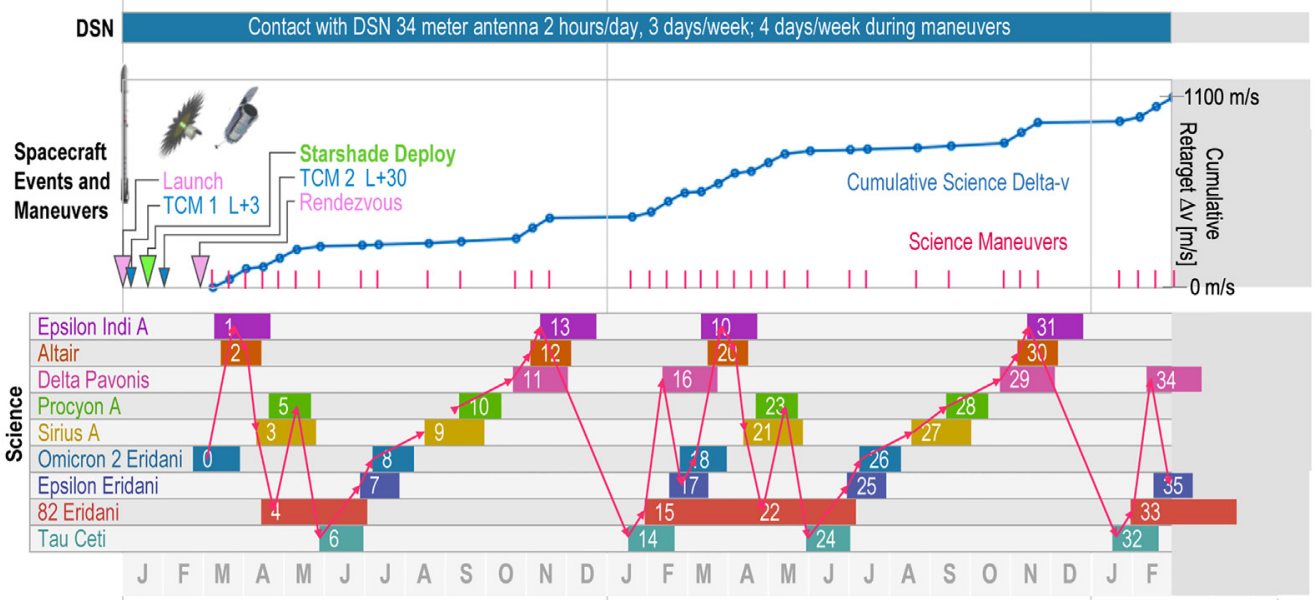

Fig. 12 An example of a retargeting strategy that bounds the fuel usage for the Starshade Rendezvous Probe mission. We have allocated 36 target maneuvers to accomplish the objectives. In this case, we have chosen a stressing case in which the 36 retarget maneuvers are applied to nine targets, each visited four times. While this is not representative of the mission, which would visit 10 habitable exoplanet targets with a subset of them requiring revisits in addition to the known gas giants, the reason this is a stressing case is that visiting a target twice a year on different availability windows requires more significant burns than having to visit a target only once a year or just once during the mission as will be that case for the known gas giants and habitability targets with large exozodiacal dust disk backgrounds. The top panel shows the main spacecraft events and maneuvers along with the cumulative $\Delta v$ estimates for each target as a function of time, labeled in year and month of year at the top of the figure. The vertical magenta tick marks show the time at which each target is visited. The bottom panel shows the targets chosen with their observing availability windows shown as horizontal bars. Each bar has numerical labels representing the order of the visits. The timing of translational retargeting slews (red lines) may take from a couple of days up to two weeks depending on the angular separation between targets. The targets are arranged vertically in order of right ascension with the observing windows for each target shown as horizontal colored bars. The observation days are chosen to be at the beginning of the window to allow time for follow-up spectroscopy within the same window. 
result in highly efficient fuel burns. With a two year window, there are cases in which fairly aggressive burns are required to catch targets of interest when their observing windows are available.

\section{Expected Performance}

The expected scientific yield depends not only on the assumed mission parameters but also on exoplanet demographics. While the frequency of gas-giant planets is relatively well known, the probability that each of our target stars will have a habitable-zone Earth-size planet $\left(\eta_{\oplus}\right)$ is not well constrained. NASA's ExoPAG SAG-13 performed a meta-analysis of several published fits to Kepler survey results, producing a planet frequency formula as a function of planet size and location. ${ }^{35}$ Combining this formula with our adopted $\mathrm{HZ}$ and Earth-like planet radius definitions (Table 6), we calculate an Earth-like planet frequency of $\eta_{\oplus}=0.24_{-0.1}^{+0.3}$. It is worth noting that this definition of Earth-like planets and corresponding frequency matches for consistency Ref. 32; for possible alternative calculations of planet occurrence rates, see, e.g., Refs. 47 and 48.

The cumulative completeness for habitability and biosignatures targets is shown in Fig. 13. The expected number of Earth-like exoplanets is derived from multiplying the completeness by the occurrence rate $\eta_{\oplus}$. For a nominal observing program targeting at least 10 nearby stars, the expected number of detected Earth-like exoplanets is $1.5_{-0.6}^{+1.9}$. However, this is only for single visit detection. The number with orbits constrained to the $\mathrm{HZ}$ is $1.2_{-0.5}^{+1.5}$. It is worth noting that the cumulative completeness for spectral measurements flattens after the fifth target. The expected number of Earth-like exoplanets with spectral characterization is $0.65_{-0.27}^{+0.82}$. The expected number with both orbits constrained and a successful spectral measurement is $0.45_{-0.19}^{+0.56}$. This number is lower than the expectation of $\sim 4$ derived by Ref. 5 for the same telescope size and starshade launch mass, primarily because Roman's actual end-to-end efficiency is lower than previously assumed, resulting in significantly longer integration times for high-quality spectra.

It is important to note that Roman observations with a starshade will be sensitive to a wide variety of planets. Figure 14 shows the expected number of planets discovered by imaging (SNR > 7), some of which are bright enough to obtain follow-up spectra. This threshold SNR value is relaxed compared with Earth-like planets because giant exoplanets tend to have more pronounced spectral features. The planet properties and frequency of occurrence are the same as the Exo-S report ${ }^{49}$ with the modification that the occurrence rate for warm Earth's and super-Earths was raised to 0.24 . These results indicate that $\sim 12$ new planets will be discovered in the nearest sunlike stars, providing additional information on their planetary system architectures.

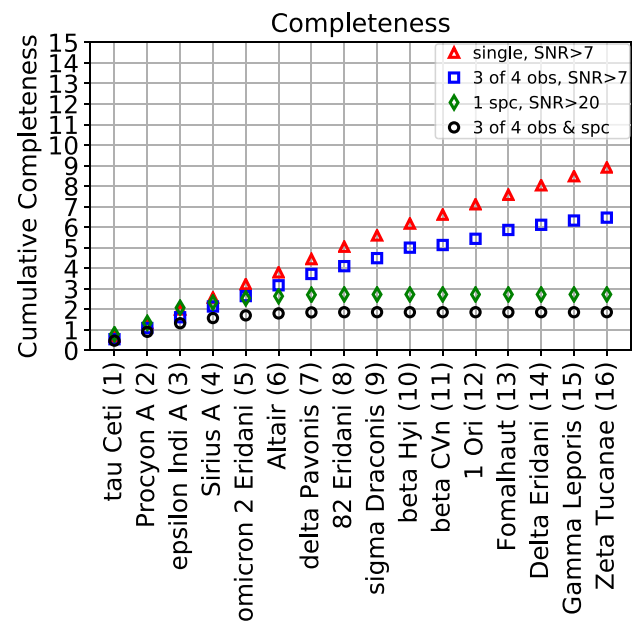

Fig. 13 Cumulative completeness for habitability and biosignatures targets (based on Fig. 8). 


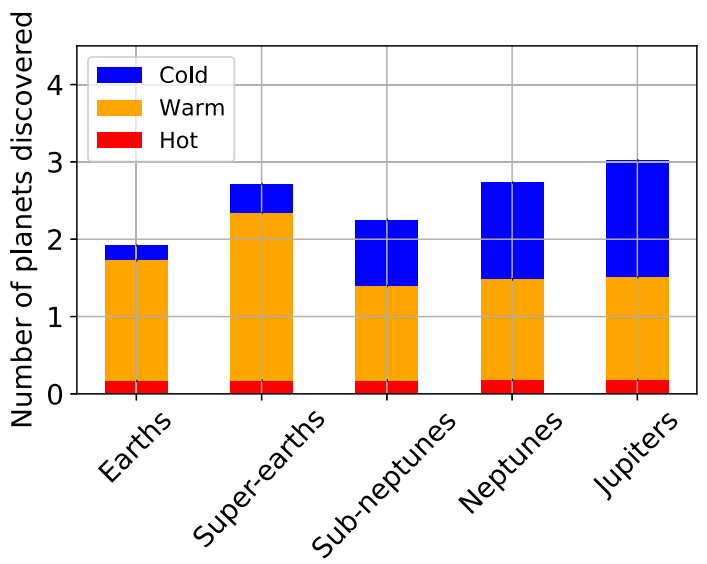

Fig. 14 Planet yield as a function of planet type and approximate temperature. The yield is obtained based on the single-visit completeness assuming detection with SNR $\geq 7$. All observations assume a zodiacal dust disk brightness of 4.5 zodi. The bar chart assumes that the 16 targets for the habitability and biosignature gases investigation are visited at least once. The number of planets scales with the number of targets visited according the the single-visit completeness curve in Fig. 13.

\section{Conclusions}

We have presented the modeling, observing approach, and expected performance to meet the objectives of the Starshade Rendezvous Probe study. ${ }^{1}$ The Starshade Rendezvous Probe concept has the capability to deliver first-of-a-kind exoplanet direct imaging and spectroscopy results in the next decade. A deep-dive investigation will provide the first examination of planetary systems around our nearest sunlike stars, including their HZs, giant exoplanets, and warm dust disks, opening a new frontier.

The Starshade Rendezvous Probe concept is capable of discovering Earth-size planets in the HZs of nearby stars using the relatively moderate aperture Roman space telescope. By initially characterizing the sensitivity to each individual target, we have found that while the SRP has the sensitivity to detect Earth-like exoplanets and constrain their orbits to the HZ, its primary limitation is the sensitivity to spectral measurements. The main means for improving this is to increase the aperture of the telescope, as would be done with HabEx, since this has the dual benefit of increasing the photon rate and better resolving the exozodiacal background. It is worth noting there are large uncertainties in the occurrence rates of Earth-like exoplanets and the distribution of zodiacal dust disk brightness, which could result in a increased discovery potential of the SRP if nature behaves favorably. The SRP is the only observatory that would have the capability to detect Earth-like exoplanets within the next decade.

Observations of known planets with the SRP could determine whether the atmospheric metallicity and mass of known giant exoplanets follow the correlation observed in our own SS, testing whether there is a trend in planetary formation. Meeting these objectives will begin to answer the driving questions of whether Earth is unique and how the SS compares with the planetary systems orbiting our nearest sunlike stars. The SRP is well equipped to meet this objective with an expected increase in the number of known gas giants with radial velocity measurements and observations with CGI prior to the SRP operations.

The SRP will obtain measurements of the exozodiacal dust of nearby sunlike stars with unprecedented sensitivity. This information is key to the future of direct imaging observatories since the dust brightness distribution is not known well enough to pin down the level of background light expected for planet detection and characterization. The sensitivity of the SRP to dust disks provides additional scientific opportunities to investigate the influence of planets on the dust disk morphology, provided such systems are found.

The main challenge of observing with starshades, retargeting with constrained time windows in a relatively short mission duration, has been addressed with a decision tree that can accommodate the large degree of uncertainty associated with searching for Earth-like exoplanets. The 
observing plan adapts to new information as the targets are observed multiple times with predetermined criteria for deciding whether to revisit targets or take spectral measurements. We have estimated that 36 retargeting maneuvers are necessary to meet the science objectives, and we have bounded the amount of delta-v needed with a stress case scenario. The driving use of fuel is the occurrence of large-angle retargeting maneuvers needed to observe some targets multiple times within the two-year duration of the mission. Depending on the realization of Earth-like exoplanet occurrence and exozodiacal dust, the SRP mission could have enough fuel for an extended mission to visit more targets.

\section{Acknowledgments}

Part of this work was carried out at the Jet Propulsion Laboratory, California Institute of Technology, under a contract with the National Aeronautics and Space Administration. (C)2020. All rights reserved. This research has made use of (1) the NASA Exoplanet Archive, which is operated by the California Institute of Technology, under contract with the National Aeronautics and Space Administration under the Exoplanet Exploration Program and (2) the SIMBAD database, operated at CDS, Strasbourg, France.

\section{References}

1. S. Seager et al., "Starshade rendezvous probe," 2019, https://smd-prod.s3.amazonaws.com/ science-red/s3fs-public/atoms/files/Starshade2.pdf.

2. M. C. Turnbull et al., "The search for habitable worlds. 1. The viability of a starshade mission," Publ. Astron. Soc. Pac. 124, 418-447 (2012).

3. D. Savransky and D. Garrett, "WFIRST-AFTA coronagraph science yield modeling with EXOSIMS," J. Astron. Telesc. Instrum. Syst. 2(1), 011006 (2015).

4. C. C. Stark et al., "Maximizing the ExoEarth candidate yield from a future direct imaging mission," Astrophys. J. 795, 122 (2014).

5. C. C. Stark et al., "Maximized exoEarth candidate yields for starshades," J. Astron. Telesc. Instrum. Syst. 2, 041204 (2016).

6. https://exoplanets.nasa.gov/exep/technology/starshade/.

7. G. Soto et al., "Starshade orbital maneuver study for WFIRST" Proc. SPIE 10400, 104001U (2017).

8. G. J. Soto et al., "Parameterizing the search space of starshade fuel costs for optimal observation schedules," J. Guidance Control Dyn. 42(12), 2671-2676 (2019).

9. W. J. Borucki et al., "Characteristics of planetary candidates observed by Kepler. II. Analysis of the first four months of data," Astrophys. J. 736, 19 (2011).

10. J. N. Winn and D. C. Fabrycky, "The occurrence and architecture of exoplanetary systems," Annu. Rev. Astron. Astrophys. 53, 409-447 (2015).

11. S. Ertel et al., "The HOSTS survey for exozodiacal dust: observational results from the complete survey," Astron. J. 159, 177 (2020).

12. H. R. Wakeford et al., "HAT-P-26b: a Neptune-mass exoplanet with a well-constrained heavy element abundance," Science 356, 628-631 (2017).

13. https://wfirst.ipac.caltech.edu/sims/Param_db.html\#coronagraph_mode.

14. https://nexsci.caltech.edu/missions/EXEP/EXEPstarlist.html.

15. K. L. Cahoy, M. S. Marley, and J. J. Fortney, "Exoplanet albedo spectra and colors as a function of planet phase, separation, and metallicity," Astrophys. J. 724, 189-214 (2010).

16. L. C. Mayorga et al., "Jupiter's phase variations from Cassini: a testbed for future directimaging missions," Astron. J. 152, 209 (2016).

17. T. D. Robinson, V. S. Meadows, and D. Crisp, "Detecting oceans on extrasolar planets using the glint effect," Astrophys. J. Lett. 721, L67-L71 (2010).

18. T. D. Robinson and C. T. Reinhard, "Earth as an exoplanet," https://ui.adsabs.harvard.edu/ abs/2018arXiv180404138R/abstract (2018).

19. T. D. Robinson et al., "Earth as an extrasolar planet: earth model validation using EPOXI Earth observations," Astrobiology 11, 393-408 (2011). 
20. C. Leinert et al., "The 1997 reference of diffuse night sky brightness," Astron. Astrophys. Suppl. Ser. 127, 1-99 (1998).

21. C. A. Beichman, N. J. Woolf, and C. A. Lindensmith, "The terrestrial planet finder (TPF): a NASA origins program to search for habitable planets," National Aeronautics and Space Administration; Jet Propulsion Laboratory, California Institute of Technology, Pasadena, California (1999).

22. B. Nemati, "Detector selection for the WFIRST-AFTA coronagraph integral field spectrograph," Proc. SPIE 9143, 91430Q (2014).

23. B. Nemati, J. E. Krist, and B. Mennesson, "Sensitivity of the WFIRST coronagraph performance to key instrument parameters," Proc. SPIE 10400, 1040007 (2017).

24. S. B. Shaklan, L. Marchen, and E. Cady, "Shape accuracy requirements on starshades for large and small apertures," Proc. SPIE 10400, $104001 \mathrm{~T}$ (2017).

25. E. J. Cady et al., "Optimal design of petal-shaped occulters for extra-solar planet detection," Proc. SPIE 6693, 669304 (2007).

26. E. Cady, "Nondimensional representations for occulter design and performance evaluation," Proc. SPIE 8151, 815112 (2011).

27. J. W. Arenberg et al., "New worlds observer system architecture," Proc. SPIE 7010, $70101 \mathrm{~S}$ (2008).

28. T. Glassman et al., "Starshade scaling relations," Proc. SPIE 7440, 744013 (2009).

29. R. Hu et al., "Overview and reassessment of noise budget of starshade exoplanet imaging," https://ui.adsabs.harvard.edu/abs/2020arXiv201201609H/abstract (2020).

30. M. C. Turnbull, "ExoCat-1: the nearby stellar systems catalog for exoplanet imaging missions," https://ui.adsabs.harvard.edu/abs/2015arXiv151001731T/abstract (2015).

31. L. A. Rogers, "Most 1.6 Earth-radius planets are not rocky," Astrophys. J. 801, 41 (2015).

32. B. S. Gaudi et al., "The Habitable exoplanet observatory (HabEx) mission concept study final report," https://ui.adsabs.harvard.edu/abs/2020arXiv200106683G/abstract (2020).

33. J. Chen and D. Kipping, "Probabilistic forecasting of the masses and radii of other worlds," Astrophys. J. 834, 17 (2017).

34. J. F. Kasting, D. P. Whitmire, and R. T. Reynolds, "Habitable zones around main sequence stars," Icarus 101, 108-128 (1993).

35. R. Belikov et al., 2017, https://exoplanets.nasa.gov/exep/exopag/sag/\#sag13.

36. C. C. Stark and M. J. Kuchner, "The detectability of exo-earths and super-earths via resonant signatures in exozodiacal clouds," Astrophys. J. 686, 637-648 (2008).

37. D. Defrère et al., "Direct imaging of exoEarths embedded in clumpy debris disks," Proc. SPIE 8442, 84420M (2012).

38. D. Sirbu et al., "Prospects for exoplanet imaging in multi-star systems with starshades," Proc. SPIE 10400, 104001D (2017).

39. A. Romero-Wolf et al., "Starshade rendezvous: exoplanet orbit constraints from multi-epoch direct imaging," Submitted (2020).

40. R. A. Brown, "Single-visit photometric and obscurational completeness," Astrophys. J. 624, 1010-1024 (2005).

41. Y. K. Feng et al., "Characterizing earth analogs in reflected light: atmospheric retrieval studies for future space telescopes," Astron. J. 155, 200 (2018).

42. F. Feng et al., "Color difference makes a difference: four planet candidates around $\tau$ ceti," Astron. J. 154, 135 (2017).

43. L. Kreidberg et al., "A precise water abundance measurement for the hot Jupiter WASP43b," Astrophys. J. Lett. 793, L27 (2014).

44. R. E. Lupu et al., "Developing atmospheric retrieval methods for direct imaging spectroscopy of gas giants in reflected light. I. Methane abundances and basic cloud properties,' Astron. J. 152, 217 (2016).

45. M. Damiano and R. Hu, "ExoReL: a Bayesian inverse retrieval framework for exoplanetary reflected light spectra," Astron. J. 159, 175 (2020).

46. E. Karkoschka, "Spectrophotometry of the jovian planets and Titan at 300- to 1000-nm wavelength: the methane spectrum," Icarus 111, 174-192 (1994).

47. D. C. Hsu et al., "Occurrence rates of planets orbiting FGK Stars: combining Kepler DR25, Gaia DR2, and Bayesian inference," Astron. J. 158, 109 (2019). 
48. S. Bryson et al., "A probabilistic approach to Kepler completeness and reliability for exoplanet occurrence rates," Astron. J. 159, 279 (2020).

49. S. Seager et al., "Exo-S report," 2015, https://exoplanets.nasa.gov/exep/studies/probe-scalestdt/.

Andrew Romero-Wolf received his BA degree in mathematics and the BA degree in physics (with honors) from the University of Chicago in 2002, his master's in physical science from the University of Chicago in 2005, and his PhD from the University of Hawaii at Manoa in 2010. He is currently a member of the technical staff at the Jet Propulsion Laboratory, California Institute of Technology, in the Deep Space Tracking group.

Biographies of the other authors are not available. 